\title{
Parsing errors in Hindi: Investigating limits to verbal prediction in an SOV language
}

\author{
Apurva, Samar Husain \\ Indian Institute of Technology, Delhi, India
}

\begin{abstract}
The role of prediction during sentence comprehension is widely acknowledged to be very critical in SOV languages. Robust clause-final verbal prediction and its maintenance have been invoked to explain effects such as anti-locality and lack of structural forgetting. At the same time, there is evidence that these languages avoid increased preverbal phrase complexity due to working-memory constraints. Given the critical role of prediction in processing of SOV languages, in this work, we study verbal predictions in Hindi (an SOV language) to investigate its robustness and fallibility using a series of completion studies. Analyses of verbal completions based on grammaticality (grammatical vs ungrammatical) as well as their syntactic property (in terms of verb class) show, as expected, frequent grammatical completions based on effective use of preverbal nouns and case-markers. However, there were also high instances of ungrammatical completions. In particular, consistent errors were made in conditions with 3 animate nouns with unique/similar case-markers. These errors increased in the face of adjuncts of differing complexity following the preverbal nouns. The grammatical and ungrammatical completions show that native speakers of Hindi posit structures with at most 2 verbal heads and 5 core verbal relations, thus highlighting an upper bound to verbal prediction and its maintenance in such configurations. A rating study confirmed that certain errors found in completion tasks can lead to grammatical illusions. Further, a detailed analysis of the completion errors in such cases revealed that the parser ignores the complete preverbal nominal features of the input and instead selectively reconstructs the input based on their frequency in the language to form illicit parses at the expense of globally consistent parses. Together, the results show that while preverbal cues are effectively employed by the parser to make clause final structural predictions, the parsing system breaks down when the number of predicted verbs/relations exceeds beyond a certain threshold. In effect, the results suggests that processing in SOV languages is susceptible to center-embeddings similar to that in SVO languages. This highlights the over-arching influence of working-memory constraints during sentence comprehension and thereby on the parser to posit less complex structures.
\end{abstract}

Keywords: Parsing error in SOV languages, Prediction fallibility, Working-memory limitation, Grammatical illusion, Good-enough processing 


\section{Introduction}

When humans process a sentence, they do not wait for it to finish in order to make sense of its meaning. Rather, they actively predict the upcoming linguistic material during the comprehension process. Experimental evidence over many decades has established this aspect of the comprehension system (e.g., Marslen-Wilson, 1973; Fischler \& Bloom, 1979; Kutas \& Hillyard, 1980, 1984; Zola, 1984; Schwanenflugel \& Lacount, 1988; Schwanenflugel \& Shoben, 1985; Altmann \& Kamide, 1999, 2007; Kliegl et al., 2004; Ashby et al., 2005; Staub \& Clifton, 2006; Rayner et al., 2011; Levy \& Keller, 2013). It is generally assumed that humans employ some form of left-corner parsing where bottom-up information is used to preactivate certain linguistic structures that in turn drive the parser in a top-down fashion (cf. Kuperberg \& Jaeger, 2016). It is not surprising, therefore, that prediction forms a critical component in many theories of language processing (Pickering \& Garrod, 2013; Pickering \& Gambi, 2018; Hale, 2001, 2006; Levy, 2008; Konieczny, 2000). The role of prediction during comprehension as well as its mechanisms form an active area of current research in psycholinguistics (Kutas et al., 2011; Petten \& Luka, 2012; Staub, 2015; Luke \& Christianson, 2016; Huettig, 2015; DeLong et al., 2014; Farmer et al., 2013).

More recently, the role of prediction has been highlighted in the processing of SOV languages (e.g., Yamashita, 1997; Koso et al., 2011; Friederici \& Frisch, 2000; Yamashita, 2000; Kamide et al., 2003; Husain et al., 2014; Levy \& Keller, 2013). In particular, increased preverbal information (including case-markers) has been shown to benefit prediction in such languages (e.g., Konieczny, 2000). Additionally, prediction is SOV languages has been shown to persist in the face of linguistic complexity (e.g., clausal embeddings). Evidence for robust prediction in SOV languages can largely be subsumed under the robust prediction and maintenance hypothesis (RPM) (Vasishth et al., 2010; Hale, 2006; Levy, 2008). The RPM hypothesis states that the parser in SOV languages becomes adept at effectively utilizing the preverbal linguistic cues to make robust clause-final verbal predictions. In addition, due to the high frequency of SOV word order, it also becomes very good at maintaining these predictions successfully. The RPM hypothesis, therefore, makes the following predictions with regard to sentence processing in SOV languages: (a) verbal prediction in SOV languages is quite robust, (b) additional preverbal material (arguments and adjuncts) make such predictions better and more precise, and (c) verbal predictions are efficiently maintained during the parsing process.

The evidence for the RPM hypothesis during online comprehension has been highlighted through behavioural, corpus, as well as computational work. One of the key effects in the behavioural literature with regard to SOV languages that has been explained using predictive processing is the so called anti-locality effect. The anti-locality effect is characterized by a processing facilitation at the verbal head with increased linear distance between the verb and its prior dependents. For example, Konieczny (2000) showed that in German sentences such as (1a), (1b), and (1c), the main verbs (hingelegt, gelegt) were read faster when the relative

Email addresses: apurvajnu@gmail.com (Apurva), samar@hss.iitd.ac.in (Corresponding author) (Samar Husain) 
clause (RC1) intervened between the verb and its argument (Er, die Rose). These results go against the memory-based accounts (e.g., Hawkins, 1994, 2014; Gibson, 1998) that predict an increase in the processing time at the verb with increase in the distance between the verb and its prior arguments. An implication of such findings is that SOV languages can allow for longer dependency lengths between verb and its argument. This tendency to allow for longer dependency lengths has been observed in cross-linguistic corpus data (Yadav et al., 2020). Anti-locality (and consequently longer dependencies) has so far been explained by two classes of theories, (a) the expectation-based information theoretic account of surprisal (Hale, 2001; Levy, 2008), and (b) the working-memory account of cue-based retrieval theory (Lewis \& Vasishth, 2005; Vasishth \& Lewis, 2006). While these accounts to anti-locality are distinct, they assume robust prediction of the verbal head.

(1) a. Er hat die Rose (RC1) hingelegt (RC2), und . . .

He has the rose (RC1) laid down (RC2), and . . .

b. Er hat die Rose (RC1) auf den Tisch gelegt (RC2), und . . .

He has the rose (RC1) on the table laid ( $\mathrm{RC} 2)$, and . . .

c. Er hat die Rose (RC1) auf den kleinen runden Tisch gelegt (RC2), und . . .

He has the rose (RC1) on the small round table laid (RC2), and . . .

Another evidence for the RPM hypothesis in SOV languages comes from the structural forgetting effect (Frazier, 1985). It has been demonstrated that native speakers of SVO languages (e.g., English, French) rate an ungrammatical sentence such as (2b) similar to its grammatical counterpart such as (2a) (Gibson \& Thomas, 1999). This grammatical illusion has been explained by assuming that English native speakers forget the prediction of the verb that incurs high memory cost (was cleaning in $2 \mathrm{~b}$ ). Interestingly, when similar sentences in German were tested with German native speakers, they failed to show such a forgetting effect (Vasishth et al., 2010). They correctly rated the ungrammatical sentence with a missing V2 ${ }^{1}$ as worse than the grammatical counterpart. This processing asymmetry between English and German has been explained by linguistic adaptability. On this account, the German native speakers are exposed to much more head-final structures in their lifetime compared to English native speakers. This makes the processing system for German better at handling head-final structures. Put simply, the expectation and maintenance of head-final structures is better in German compared to English. Such lack of structural forgetting has also been reported in a language like Dutch (Frank et al., 2016). Under such parsing assumptions, Futrell et al. (2020) have successfully modelled this English vs German processing difference.

(2) a. The apartment that the maid who the service had sent over was cleaning every week was well decorated.

b. * The apartment that the maid who the service had sent over $\emptyset$ was well decorated.

\footnotetext{
${ }^{1}$ Note the syntactic structure in example 2a is of the form [N1 [N2 [N3 V3] V2] V1], with N1 being syntactically related to V1, N2 to V2 and so on. In example 2a, V1 corresponds to decorated, V2 corresponds to was cleaning and V3 corresponds to had sent over.
} 
To summarize, the RPM hypothesis is supported by anti-locality and no structural forgetting effects in SOV languages, in addition to corpus-based studies demonstrating presence of longer dependency length in SOV languages. Other works using the visual-world paradigm (e.g., Kamide et al., 2003), eye-tracking corpus study (e.g., Boston et al., 2008, 2011; Husain et al., 2015; Agrawal et al., 2017) have also highlighted the crucial role of prediction in SOV languages. Importantly, these accounts assume a parsing process that does not lead to globally illicit parses in SOV languages due to prediction fallibility. From a typological perspective, these processing results in SOV languages can be contrasted with results from SVO languages (e.g. English) where evidence for locality and forgetting effect has been demonstrated (e.g., Grodner \& Gibson, 2005; Gibson \& Thomas, 1999). Consequently, a differential processing account based on the typological word order feature of a language has been posited (e.g., Vasishth et al., 2010).

Notwithstanding, the evidence for prediction in SOV language, there is also evidence that predictive processing interacts with working-memory constraints. For example, Vasishth \& Drenhaus (2011) showed that in German similarity-based interference amongst preverbal nominals can lead to locality effects. Similarly, Vasishth \& Lewis (2006) showed that increased similarity between preverbal nominals can nullify the anti-locality effect in Hindi. Levy \& Keller (2013) showed that increased dependency distance while building a complex structure can lead to locality effects in German. While these works have focused on the retrieval processes at the verb, it remains a possibility that in such configurations verbal predictions made prior to the actual verb suffers. For example, Bhatia \& Husain (2018, 2020) have shown that in Hindi, a non-canonical word order in a clausal embedding can lead the parser to forget the prior prediction of the matrix verb leading to constructions of illicit parses during online processing. This suggests that increased working memory load due to structural complexity can lead to prediction fallibility in SOV languages. Such a fallibility would lead to processing difficulty in such syntactic configurations. Similar proposals have been made by Ueno \& Polinsky (2009); Gibson et al. (2013); Ros et al. (2015). For example, Ueno \& Polinsky (2009) found evidence for a pro-drop and intransitive bias in SOV languages (also see Harinuma, 1999). Using a corpus study they argued that pro-drop and frequent use of intransitives is a strategy in SOV languages to reduce the dependency length between the clause final verb and its prior arguments. In a eye-tracking corpus study, Husain et al. (2015) demonstrated the cost of maintaining multiple syntactic dependency heads (computed using DLT's storage cost metric, see Gibson, 1998) on various eye-movement measure. Relatedly, in a recent large-scale cross-linguistic corpus study, Yadav et al. (2020) suggest that the tendency to have long dependency distance in SOV languages is substantially reduced in the case of complex syntactic construction (e.g., increased complexity of the intervening phrases between the verb and its arguments, discontinuous phrases).

Predictive processing in SOV languages has been frequently pitted against workingmemory constraints because effects such as anti-locality and lack of structural forgetting go against the assumption that complex syntactic configurations should lead to processing difficulty. The research discussed in the previous paragraph provides compelling evidence that working-memory constraints can modulate various structural properties in SOV languages. An implication of this research is that verbal predictions in SOV languages could 
suffer in the face of configurations that make the linguistic environment prior to the verb complex. We call this the Fallible prediction and maintenance (FPM) hypothesis. The FPM hypothesis predicts that while prediction in SOV languages is robust, it is also constrained by working-memory pressures. In particular, it predicts that parsing errors due to incorrect verbal prediction will increase in cases such as increased structural depth, etc. Indeed, as we discuss later, the FPM hypothesis is compatible with comprehension accounts that do not assume an error free parsing process (e.g., Ferreira \& Patson, 2007; Gibson et al., 2013; Kurumada \& Jaeger, 2015). From a typological perspective, such an account posits that both SVO and SOV languages are susceptible to working-memory constraints due to increased linguistic complexity. For example, one prediction would be that presence of center-embedded structures will lead to parsing errors in both SOV and SVO languages.

Given the proposed wide-ranging role of prediction in processing of SOV languages, it is critical to evaluate the robust prediction and maintenance (RPM) hypothesis and the fallible prediction and maintenance (FPM) hypothesis rigorously. To our knowledge, such an investigation is currently lacking. When does the parsing process fail due to prediction fallibility? And more importantly, if the parser leads to an erroneous parse, then what is the nature of the incorrect parse? In this work, we conduct a series of sentence completion and rating studies to investigate these questions.

The paper is arranged as follows. In section 2, we present a pilot sentence completion study where verbal prediction based on differing number of preverbal nouns and case-markers is investigated. The results of the pilot study is then used to investigate the role of casemarker order variation and case-marker similarity on prediction. This study is discussed in section 3. The completion study in section 4 investigates how verbal predictions are maintained when additional preverbal adjuncts are introduced. Finally, in Section 5, a rating study in reported to investigate the acceptability of correct and incorrect predictions reported in section 3. We consolidate the findings from experiments 1-4 and discuss its implications in Section 6. We conclude the work in section 7.

\section{Experiment 1}

This section reports a pilot sentence completion study that investigates the role of the number of preverbal nouns and their case-markers on clause-final verbal prediction. This study was undertaking to understand the larger prediction trends and was used to inform the designs of experiments 2 and 3.

\subsection{Material and methods}

\subsubsection{Participants}

25 native speakers of Hindi participated in the experiment. All the participants were undergraduate or graduate students from the Indian Institute of Technology, Delhi. The average age of the participants was 20.84 years (SD:3.17). Each participant was paid INR 200 for participating in the experiment. 


\subsubsection{Items}

We investigated the role of the number of preverbal nouns (NP) and their case-markers on clause-final verbal prediction. The number of nouns ranged from 1 to 3 . The casemarkers on the nouns were $\emptyset$, 'ne', 'ko', or 'se'. $\emptyset$ stands for the Nominative case, 'ne' is the Ergative case, 'ko' is the Accusative case and 'se' is Ablative/Instrument case. ${ }^{2}$. The preverbal nouns used in the items were always proper nouns (either male human or female human). All combinations of preverbal nouns and case-markers were constructed. There were 4 conditions with a single preverbal noun (1-NP conditions), each corresponding to the 4 case-markers mentioned above. There were 15 conditions with two preverbal nouns (2-NP conditions). And there were 52 conditions with three preverbal nouns (3-NP conditions). In all, the experiment had 71 conditions. Example 3a-j shows some of these conditions.

$$
\begin{aligned}
\text { a. } & 1-\mathrm{NP}, \emptyset \\
& \text { rahul } \ldots \\
& \text { Rahul . . } \\
\text { b. } & 1-\mathrm{NP}, \text { ne } \\
& \text { rahul-ne } \ldots \\
& \text { Rahul-ERG ... } \\
\text { c. } & 1-\mathrm{NP}, \text { ko } \\
& \text { jayant-ko } \ldots \\
& \text { Jayant-ACC ... } \\
\text { d. } & 1-\mathrm{NP}, \text { se } \\
& \text { sita-se } \ldots \\
& \text { Sita-ABL ... } \\
\text { e. } & \text { 2-NP, ne-ko } \\
& \text { prakash-ne ritu-ko } \ldots . \\
& \text { Prakash-ERG Ritu-ACC ... }
\end{aligned}
$$

f. 2-NP, ko-ko

$$
\begin{array}{ll}
\text { ajay-ko lata-ko } & \ldots \\
\text { Ajay-ACC Lata-ACC } . .
\end{array}
$$

g. 2-NP, se- $\emptyset$

jayant-se kapil ...

Jayant-ABL Kapil ...

h. 3-NP, ne-ko-se

pooja-ne urmila-ko suneet-se ...

Pooja-ERG Urmila-ACC Suneet-ABL ...

i. $3-\mathrm{NP}$, ne-ko-ko

\footnotetext{
${ }^{2}$ Case-markers in Hindi can be ambiguous. For example, 'ko' could be interpreted either as Accusative or Dative; although, the default reading is Accusative. However, the 'ne' case-marker is unambiguously Ergative. See Kachru (2006), for an overview of case-markers in Hindi.
} 


$$
\begin{array}{lllll} 
& \text { ajay-ne lata-ko dileep-ko } & \ldots \\
& \text { Ajay-ERG Lata-ACC Dileep-ACC ... } \\
\text { j. } & 3-N P, \text { ne-se-ko } \\
& \text { ruchi-ne sita-se hari-ko } \quad . . \\
& \text { Ruchi-ERG Sita-ABL Hari-ACC ... }
\end{array}
$$

12 items were prepared for all conditions. Each item and its conditions were presented to the participants using a latin-square design based on item id. In all 12 lists were prepared.

\subsubsection{Procedure}

The sentence completion task was employed as the experimental paradigm (Taylor, 1953). Each sentence was presented using the centered self-paced reading (SPR) paradigm. Participants were provided an incomplete sentence and their task was to complete it such that it was meaningful. Each sentence appeared on the screen in the self-paced reading format. Initially, a ' + ' sign appeared on the center of the computer screen. When the participant pressed the space-bar key this + sign got replaced with the first word of the sentence. Successive button presses displayed the remaining words of the sentence at the center of the screen. A ... symbol prompted the participant to complete the sentence. This was done in a text box that appeared by pressing 'space-bar' after the ... symbol. After typing in the text the participants pressed the 'enter' key to move to the next trial. The experiment was conducted using Douglas Rohde's Linger software ${ }^{3}$. Items were automatically randomized by Linger.

The sentence completion task is the go-to paradigm to quantify predictability during comprehension. Previous studies on word predictability employing the sentence completion task have shown that completion patterns correlate strongly with reading time patterns found during online sentence comprehension (Levy \& Keller, 2013; Jäger et al., 2015; Husain et al., 2014; Rayner et al., 2011). Indeed, it has been argued that cloze probabilities from the completion task provide the most comprehensive measure to quantify predictability (Staub, 2015). The correlation between completion and comprehension patterns has been shown at different granularity of completion results, for example, for prediction of exact lexical item (Kliegl et al., 2006; Husain et al., 2014; Jäger et al., 2015; Lowder et al., 2018), morphosyntactic information (Dikker et al., 2009, 2010; Luke \& Christianson, 2015), prediction of verb-class (Levy \& Keller, 2013) as well as for prediction of semantically related words (McRae et al., 1998; Luke \& Christianson, 2016). See Staub et al. (2015); Staub (2015); Luke \& Christianson (2016), for an extensive overview.

\subsubsection{Response coding}

After running the experiment, participant responses were coded for the following information: (a) Grammaticality: grammatical completions were coded as 1 and ungrammatical completions were coded as 0; (b) Verb class: transitive verbs were coded T, intransitives IN, ditransitives DT, experiencer verbs as EXP, copula as COP and causatives as CAUS. Both

\footnotetext{
${ }^{3}$ version 2.94
} 
the grammaticality and verb class coding was done based on the predicted matrix verb. The coding was done by the first author; this coding was subsequently validated by a second coder.

The decision to analyze the completion data for verb type (intransitive, transitive, etc.) rather than for exact lexical information was made to help us capture the relationship between the preverbal nouns and the properties of the upcoming verb. It is known that the number of preverbal nouns, their case-markers and semantics, constrain the properties of the upcoming verbs in Hindi (similar to other SOV languages). As expected, these constraints act both at the level of subcategorization as well as certain syntactic features. For example, use of the Ergative case-marker ensures that the upcoming verb has to be transitive with a perfective aspect. Other completions will make the sentence ungrammatical. ${ }^{4}$ Such constraints hold for other case-marker combinations (see Kachru (2006), for an overview of case and verbal properties in Hindi). Not surprisingly, previous work has shown that cloze probability of verb class information correctly predicts online comprehension pattern (Levy \& Keller, 2013). The decision to analyze verb class was also guided by recent results where graded predictions (predictions related to category, semantics, morpho-syntactic information) have been shown to be capturing predictability better than all-or-nothing (lexical) prediction (Staub, 2015; Luke \& Christianson, 2016).

\subsection{Predictions}

The RPM hypothesis predicts robust predictions of clause final matrix verbs based on the prior nominal properties. It is therefore expected that if an incomplete sentence has a meaningful completion possible, then it must be completed grammatically by a Hindi native speaker. On the other hand, the FPM hypothesis predicts that verbal predictions will suffer in the face of complex configurations. These configurations could involve non-canonical word order, similar case-markers on nouns, and positing of embeddings in order to make valid grammatical prediction.

\subsection{Results}

The results of the pilot study will be largely descriptive in nature. Wherever applicable, the statistical analysis for grammaticality has been done using the generalized linear mixedeffects model with logit link function. This has been done using the lme4 package (Bates et al., 2015) in R. Maximal models were fit when possible (Barr et al., 2013); in case of convergence failure, a less complex model was fit by successively removing the random slopes of the by-subject and by-item random effects component. We first discuss the results on grammaticality and then turn towards the verb class results. ${ }^{5}$

\footnotetext{
${ }^{4}$ There are some exceptions to this pattern. For example, the subjects of certain classes of intransitive verb can also bear an Ergative case-marker. However, such usage is rare compared to the transitive verb usage.

${ }^{5}$ All the data and analysis files for experiments 1-4 can be downloaded from https://osf.io/nj5rt/
} 


\subsubsection{Grammaticality}

Results show that the overall percentage of grammatical completions across all items was $51 \%$ (see figure 1). Around $17.5 \%$ of the completions were completed ungrammatically. The remaining $31.4 \%$ were completions that can be interpreted as grammatical assuming an elision of a coordinating conjunct. For example, 'N1-0 N2-0' can be treated as 'N1-0 and N2-0' while making a prediction. Such coordinating conjunct can be elided in natural speech. Wherever a completion can be deemed grammatical by assuming such devices, we code it 'ambiguous' as these completions could also be ungrammatical if the participant did not assume a coordinating conjunct. It is quite possible that some of these are errors, but there is no way for us to ascertain that. For all analyses of ungrammatical completions in this experiment and later experiments, we do not consider such ambiguous completions. Therefore, the ungrammatical percentage of $17.5 \%$ is quite conservative and can be treated as a lower-bound.

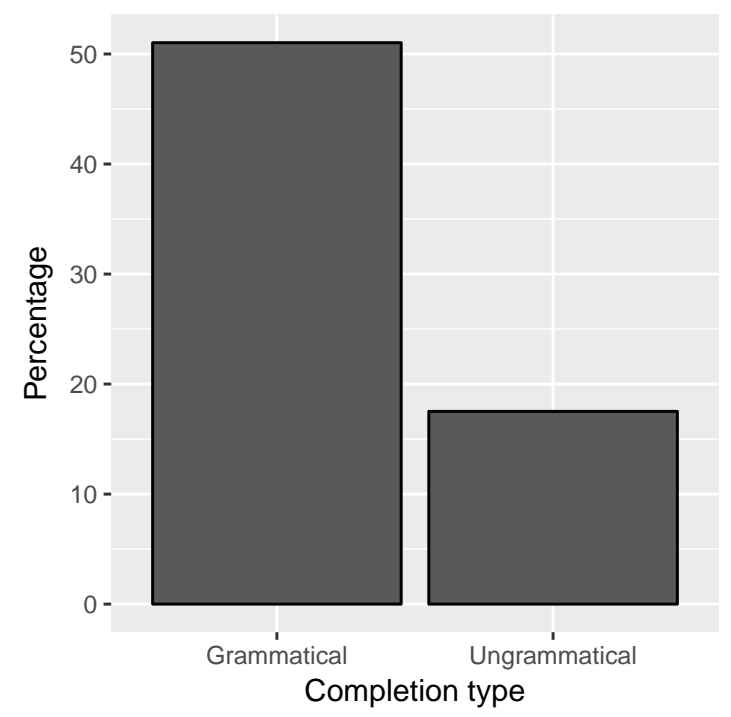

Figure 1: Average grammatical vs ungrammatical completions across all items.

A closer look at the ungrammatical completion data based on the number of preverbal nouns shows that, as expected, the completion accuracy was quite high in the 1-NP conditions. The percentage of ungrammatical completions in the 1-NP conditions was $4 \%$ (i.e., around $96 \%$ of 1 -NP conditions were completed grammatically). In the 2 -NP conditions, the percentage ungrammatical completions was around $8 \%$. However, this percentage increased drastically in the 3 -NP conditions to $22 \%$ (only around $36 \%$ of all $3-\mathrm{NP}$ conditions were completed grammatically). This can be seen in Figure 2.

Figure 3 shows all the 2-NP conditions and some 3 -NP conditions where the percentage of ungrammatical completion was relatively high $(>10 \%)$. Interestingly, in both 2-NP cases as well as 3-NP cases, conditions with high percentage of ungrammatical completion had more than one identical case-markers. For example, in the 2-NP cases, such conditions were N1ko N2-ko, N1-se N2-se, etc. Similarly, in the 3-NP cases, high ungrammatical completions 


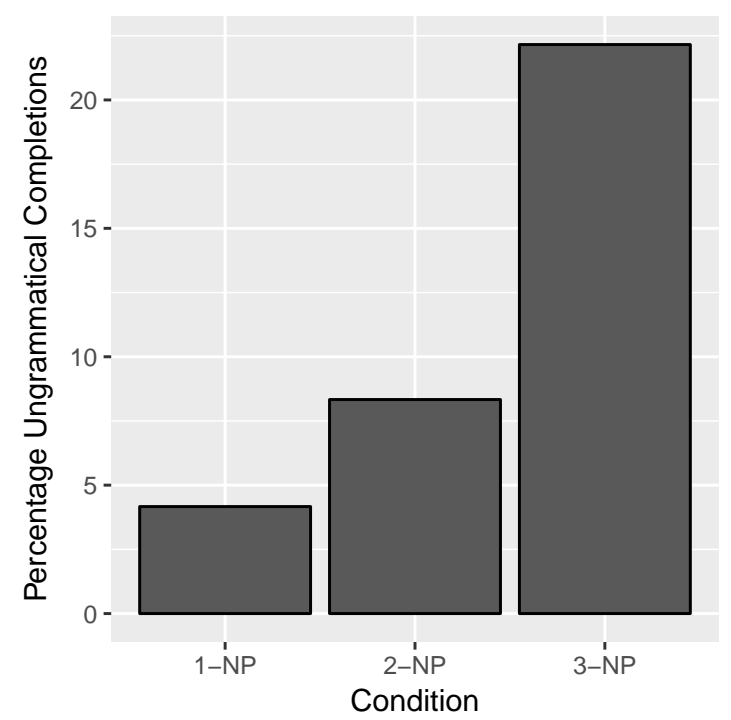

Figure 2: Percentage ungrammatical completions in conditions 1-NP, 2-NP and 3-NP.

were observed in N1- $\emptyset \mathrm{N} 2$-ne N3- $\emptyset$, N1-se N2-ne N2-se, etc. In addition to conditions with multiple identical case-markers, high ungrammatical completions in the 3-NP conditions were also observed in conditions with non-canonical order of case-marker, e.g., N1-ko N2-se N3- $\emptyset$, N1-se N2- $\emptyset$ N3-ko, etc.
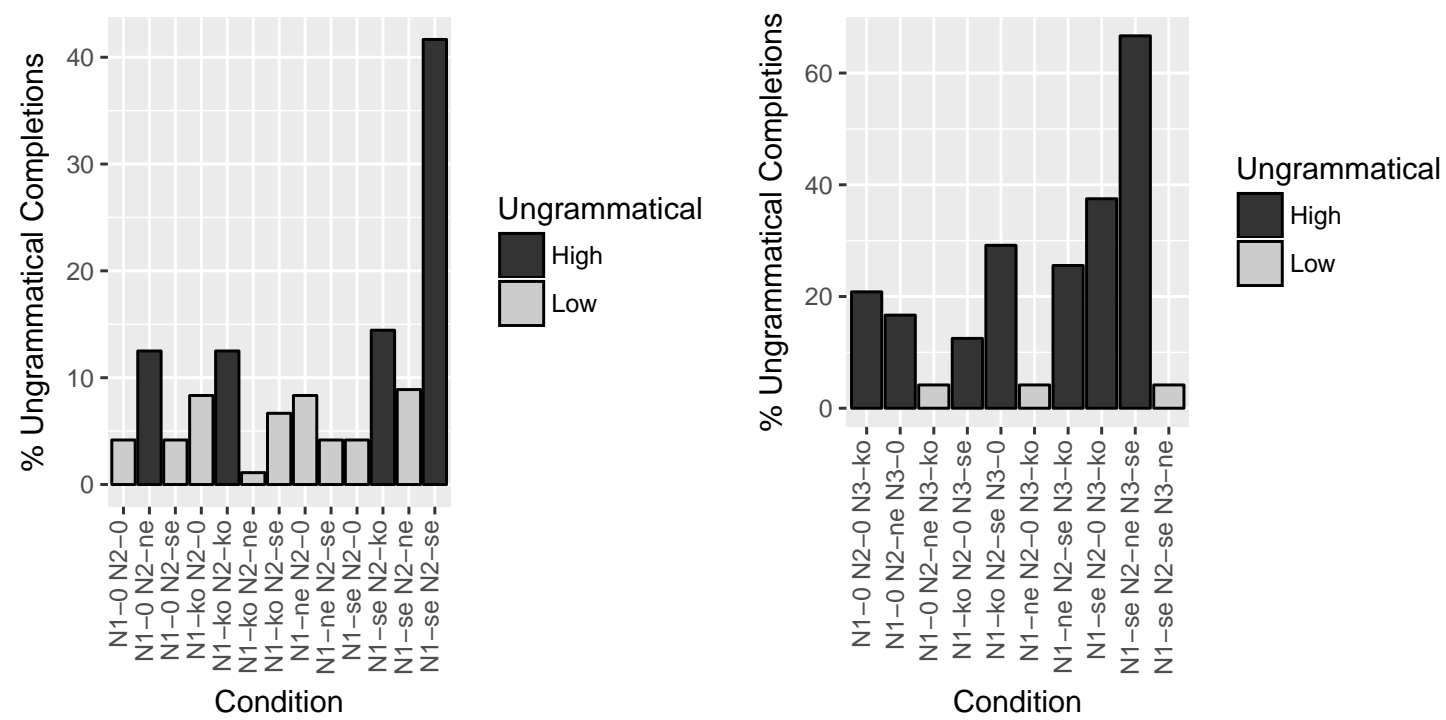

Figure 3: Percentage grammatical and ungrammatical completions in the 2-NP and 3-NP conditions. 'High' (dark grey bars) represents more than $10 \%$ ungrammatical completions, 'Low' (light grey bars) represents less than $10 \%$ ungrammatical completions. 


\subsubsection{Verb Class}

We now discuss the results of the completion study based on the predicted verb class of the matrix verb. Here, we only consider those completions that were grammatical. As stated earlier, the following verb classes were coded for the completion data: intransitive verbs, copular verbs, experiencer verbs, transitive verbs, ditransitive verbs and causative verbs.

In the 1-NP conditions most of the verbs were intransitive, copular or experiencer. One exception was the N1-ne condition which was completed with transitive verbs ${ }^{6}$. In the 2-NP condition the most dominant verb class was transitive. The most frequent verb classes in the 3 -NP condition were ditransitives, causative and transitives. These results were on expected lines and reflect the constraints on the argument structure of verbs in different classes. This can be seen in Figure 4.

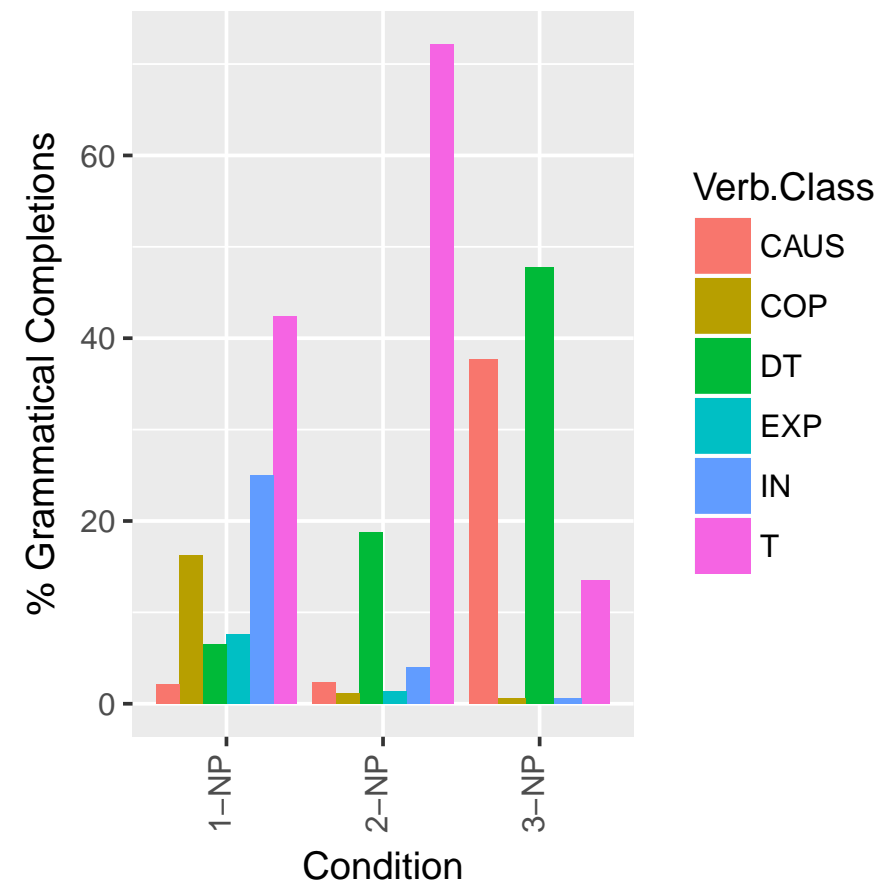

Figure 4: Percentage grammatical completions in the 1-NP, 2-NP and 3-NP conditions for various verb classes. CAUS: Causative, COP: Copula, DT: Ditransitive, EXP: Experiencer, IN: Intransitive, T: Transitive.

\subsubsection{A preliminary analysis of parsing errors}

As stated above, the 3-NP conditions form the bulk of parsing errors due to incorrect predictions. This includes conditions with both unique case-markers as well as conditions with identical case-markers. But what is the nature of the errors in such 3 -NP conditions?

\footnotetext{
${ }^{6}$ As stated earlier, the most frequent appearance of the Ergative case-marker (ne) in Hindi is with a transitive verb with a perfective aspect. In the N1-ne condition, the participants end up positing an additional noun in order to satisfy the transitivity constraint of the predicted verb.
} 
A closer look at the error patterns in such cases suggest that the participants tend to make a variety of very systematic errors. In one type of error, the first noun (N1) of the sentence fragment is ignored to make the completions (see Table 1). For example, an instance such as N1-ko N2-ne N3-ko (pujaa ko urmila ne sunitaa ko) was completed with a transitive verb dekha 'see'. This completion would have been correct if one ignores N1-ko. In all, close to $14.2 \%$ completions out of the total completions were ungrammatical because of this reason. In the second type of error, the case-marker of one of the nouns was misinterpreted. For example, in Table 1, pujaa ko urmila ne sunitaa ko was completed with a causative verb milwaayaa 'meet-caus'. This completion would have been correct if one assumes N1-ko to be $N 1$-se. In all, close to $3.1 \%$ completions out of the total completions were ungrammatical because of this reason. Given the nature of this experiment, we refrain from elaborating on these and other error types further. The detailed analysis of various error types will be taken up in Experiment 2.

\begin{tabular}{|c|c|}
\hline Condition & Completion \\
\hline XN1 1 K & $\overline{\mathrm{T}}$ \\
\hline pujaa ko urmila ne sunitaa ko & nahi dekhaa \\
\hline 'Pooja $=$ ko Urmila $=$ ne Sunita $=$ ko' & 'not see' \\
\hline N1-kł фse N2-ne N3-ko & CAUS \\
\hline pujaa ko urmila ne sunitaa ko & milwaayaa \\
\hline 'Pooja $=$ ko Urmila $=$ ne Sunita $=$ ko' & 'meet-CAUS' \\
\hline
\end{tabular}

Table 1: Ungrammatical completions for some conditions with similar case-marker combination.

\subsection{Discussion}

The results of the pilot study show four clear patterns, (a) no. of preverbal nouns and their case-markers are being effectively employed to make correct verbal prediction, (b) prediction of clause-final verbs in an SOV language like Hindi can be ungrammatical, (c) increase in the number of preverbal nouns can make prediction worse, and (d) presence of nouns with identical case-markers and non-canonical case-marker order leads to increase in ungrammatical predictions.

The results of the grammaticality analysis are quite striking. Close to $17.5 \%$ of the completions were ungrammatical. This percentage is quite high considering that all the 71 conditions can in fact be completed grammatically. The majority of these errors happen in the 3-NP conditions. It is worth noting here that a sentence fragment involving 3-NPs can be completed grammatically in a variety of ways. Table 2 shows some possible continuations for $N 1=n e N 2=k o N 3=s e \ldots$ sentence fragment ${ }^{7}$. This fragment can either be completed by positing a causative verb or by positing multiple verbs involving center embeddings. Table 2 also shows the number of embeddings and the number of (explicit and implicit) core verbal relations needed for possible verb combinations. We find that use of multiple verbs

\footnotetext{
${ }^{7}$ Note that $N 1=n e N 2=k o N 3=s e \ldots$ presents a canonical order of these case-markers.
} 
involves around 2-3 clauses and around 5-9 syntactic relations leading to multiple unresolved dependencies prior to the verb. For example, if an intransitive non-finite verb (IN.NFV) is posited after $\mathrm{N} 3=\mathrm{se}$, it will require a prediction of two additional verbs - a ditransitive non-finite verb (DT.NFV) and a ditransitive matrix verb (DT). This will lead to a doubly center-embedded structure. The 3-NP fragment can be completed with a single matrix verb involving a causative. While the use of causatives involves a single clause and just 3 relations, they are quite rare in the language ${ }^{8}$, presumably due to encoding a very complex event structure in addition to having a complex morphology. This suggests that predicting and maintaining the structures in both causative as well as multiple verbs options with multiple embeddings and unresolved dependencies, should involve considerable processing load and should strain the working-memory. Indeed, such configurations are known to incur processing load during comprehension (Yngve, 1960; Miller \& Chomsky, 1963; Kimball, 1973; Frazier, 1985; Wu et al., 2010; Gibson, 1998; Lewis, 1993). This could be the reason for the high completion errors in the 3-NP conditions which form the bulk of parsing errors due to incorrect predictions. In light of this, the results support the FPM hypothesis and highlight the influence of working-memory constraints on parsing.

The verb class results for grammatical completions do not throw up many surprises. The dominant verb classes used in the 1-NP conditions were intransitive, experiencer and copular. Similarly, in the 2-NP conditions transitives dominated the completions. In the 3-NP conditions, the majority of verbs belonged to the ditransitive and causative verb class. This follows from our knowledge about the subcategorization requirements of these verb classes in terms of the number, type of the nouns as well as their case-markers. These patterns are quite reassuring and partly support the RPM hypothesis - Hindi native speakers do effectively use such nominal cues for verbal predictions in various conditions.

To summarize the completion results based on grammaticality, we find that prediction in an SOV language like Hindi is fallible, leading to certain systematic parsing errors. Predictions are quite good when the no. of preverbal nouns are less and the case-markers are distinct. At the same time, parsing errors increase for 3-NP conditions for both canonical and non-canonical case-marker order with unique case-markers. Errors are higher when case-markers are repeated. The results support the FPM hypothesis and highlight fallibility in prediction during comprehension due to increased processing load in the 3-NP conditions.

The pilot study provided a broad outline regarding the nature of verbal prediction in Hindi. We next discuss two experiments that aim to understand the parsing error types more thoroughly. Since the 3-NP conditions lead to most errors, the next experiment focuses on these. In particular, we use the 3-NP conditions with unique case-markers in canonical and non-canonical case-markers. In addition, we use the similar case-marker patterns in the 3-NP conditions where these case-markers are non-adjacent.

\footnotetext{
${ }^{8}$ In a Hindi dependency treebank (Bhatt et al., 2009), out of all the finite verb instance, there are just $0.02 \%$ of causative verbs.
} 


\begin{tabular}{|l|c|c|}
\hline & $\begin{array}{c}\text { No. of verbs/ } \\
\text { clauses }\end{array}$ & No. of core relations \\
\hline & & \\
\hline
\end{tabular}

Table 2: Possible structures to complete N1=ne N2=ko N3=se sentence fragment with various verb types. Underlined text represents possible completions. Round braces represent clausal boundaries. Green arcs represent syntactic relations between the matrix verb and its arguments. Black arcs represent syntactic relations between non-finite verb and its argument. Dotted red arcs represent implied arguments. Blue arc represents adjunct relation between the finite verb and the non-finite verb. 'ne': Ergative case-marker, 'ko': Accusative case-marker, 'se': Ablative case-marker. CAUS: Causitive matrix verb, DT: Ditransitive matrix verb, T.NFV: Transitive non-finite verb, IN.NFV: Intransitive non-finite verb, DT.NFV: Ditransitive non-finite verb. 


\section{Experiment 2}

Results from the pilot study suggests that use of 3 preverbal proper nouns leads to increased parsing errors in Hindi. In order to investigate verbal predictions in such cases more thoroughly, in this section we report two separate experiments.

Experiments $2 \mathrm{a}$ and $2 \mathrm{~b}$ respectively focus on (a) the order of unique case-markers for 3 preverbal nominals, and (b) presence of non-adjacent similar case-markers, again with 3 preverbal nominals. In case of similar case-markers, non-adjacency is important. Recall that many completions, in conditions with adjacent nouns with similar case-markers (e.g., N1-ne N2-se N3-se ... ), can be treated as grammatical by assuming coordination of adjacent nominals. In order to avoid such ambiguous interpretations, we only employ non-adjacent case-marker patterns for Experiment 2b.

\subsection{Material and methods}

\subsubsection{Participants}

36 native speakers of Hindi participated in this experiment. These subjects did not participate in any other experiment discussed in the paper. All the participants were undergraduate or graduate students from the Indian Institute of Technology, Delhi. The average age of the participants was 24.3 (SD=3.08). Each participant was paid INR 150 for participating in the experiment.

\subsubsection{Items}

We first discuss the items for unique case-markers (Experiment 2a) followed by nonadjacent similar case-markers (Experiment 2b). The unique case-marker conditions are shown in sentence 4. Each condition had 3 proper nouns with Ergative 'ne', Accusative 'ko' or Ablative 'se' case-marker in different order. This led to 6 conditions.
a. ne-ko-se
pooja-ne urmila-ko suneet-se $\ldots$
Pooja-ERG Urmila-ACC Suneet-ABL ...
b. ne-se-ko
pooja-ne urmila-se suneet-ko ...
Pooja-ERG Urmila-ABL Suneet-ACC
c. ko-ne-se
Pooja-ACC Urmila-ERG Suneet-ABL
pooja-ko urmila-ne suneet-se ...
d. ko-se-ne
pooja-ko urmila-se suneet-ne ...
Pooja-ACC Urmila-ABL Suneet-ERG
e. se-ko-ne
pooja-se urmila-ko suneet-ne ...
Pooja-ABL Urmila-ACC Suneet-ERG 


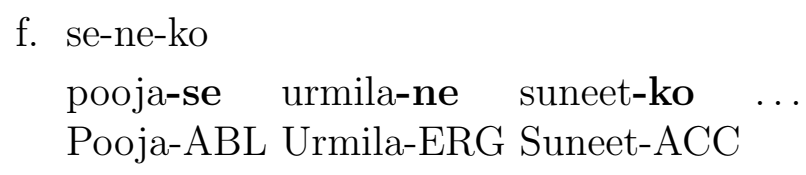

For the similar case-marker experiment, only the Accusative and Ablative case-markers were repeated. This is because a sentence fragment with two non-adjacent Ergative casemarkers cannot be completed grammatically. These items are shown in sentence 5
a. ko-ne-ko
pooja-ko urmila-ne suneet-ko ...
Pooja-ACC Urmila-ERG Suneet-ACC
b. ko-se-ko
pooja-ko urmila-se suneet-ko
Pooja-ACC Urmila-ABL Suneet-ACC
c. se-ne-se
pooja-se urmila-ne suneet-se ...
Pooja-ABL Urmila-ERG Suneet-ABL
d. se-ko-se
pooja-se urmila-ko suneet-se $\ldots$
Pooja-ABL Urmila-ACC Suneet-ABL

12 items with the different conditions were constructed for both unique case-marker and similar case-marker experiments. In addition, 60 filler items were prepared. The filler items comprised of different construction types. These were, embedded relative clauses, correlative constructions, sentences with two proper nouns with differing case-markers, sentences with two proper nouns and an adverbial/inanimate noun with differing case-markers, sentences with two pronouns, declarative sentences with non-canonical word order, other random sentences taken from a news corpus. The items of the two experiments along with the filler were presented using the latin-squared design.

\subsubsection{Procedure}

Similar to Experiment 1, we follow the sentence completion paradigm. See section 2.1.3 for details.

\subsection{Response coding}

Similar to Experiment 1, response coding was done for grammaticality of the completion as well as the verb type. Grammaticality was coded as 0 if the completion was ungrammatical and 1 if it was grammatical. In addition, certain completions that were completed assuming coordination of the first two nouns were coded as 2. In such completions, participants coordinated the first 2 nouns with the third noun and another noun used during completions. For example, an N1-ko N2-ne N3-ko condition was treated a N1-ko N2-ne and N3-ko N4-ne. N4-ne signifies an additional noun/pronoun with an Ergative case-marker posited during the 
completion task. For the analysis discussed later, we remove all such completions. This led to removal of $7.7 \%$ of the data.

Response coding was also done for verb class of completion. Coding was done such that 3 nouns in the sentence fragments were interpreted as arguments of the verb(s) used to complete a condition. We analyse all such non-finite and matrix verbs used in the completion data. Coding was done for the following verb class: Transitive: T, Intransitive: IN, Ditransitive: DT, Experiencer: EXP, Copula: COP and Causatives: CAUS. For example, if the completion had one transitive non-finite verb and a ditransitive matrix verb, then it was coded as T+DT. Note that if the participants used additional nouns during completions, they were coded only if they formed part of the argument structure of the verbs. The coding was done by the first author; this coding was subsequently validated by a second coder.

\subsection{Predictions}

The RPM hypothesis will predict robust prediction for the two sub-experiments. As noted earlier, these case-marker combinations can lead to grammatical completions. The RPM hypothesis predicts effective usage of the nominal features to predict the required number of verbs. In particular, it does not predict use of globally illicit parses in SOV languages due to prediction fallibility. The FPM hypothesis, on the other hand, will predict high error rates in various 3-NP patterns as they require positing complex structures (cf. Table 2). In addition, higher error rates are predicted in the similar case-marker conditions. Note, however, since the 3-NP conditions have been shown to have increased occurrence of parsing errors in the previous experiment, the key aim of the two experiments was to study the nature of these parsing errors.

\subsection{Results}

All the statistical analysis for the grammaticality of completions was done using the generalized linear mixed-effects model with logit link function. This has been done using the lme4 package (Bates et al., 2015) in R. Maximal models were fit when possible (Barr et al., 2013); in case of convergence failure, a less complex model was fit by successively removing the random slopes of the by-subject and by-item random effects component.

Verb class errors were also analyzed using the method discussed above. These errors were divided into the following types, (a) N1-N2: when the first two nouns were used to predict the verbs, (b) N2-N3: when the last two nouns were used to predict the verbs, (c) N1-N3: when the first and the last nouns were used to predict the verbs, (d) Case-exchange: when one of the case-markers on N1, N2 or N3 was misinterpreted to predict the verbs, (e) Random: when errors could not be classified as (a)-(d).

\subsubsection{Results: Unique case-marker (Experiment 2a)}

Results for the grammaticality analysis show no difference between the canonical word order condition vs the other non-canonical conditions (see, Table 3). The average parsing error across all condition due to incorrect prediction was around $15 \%$. This can be seen in 
Figure 5. Futher, a comparison of these 3-NP conditions with 2-NP filler items ${ }^{9}$, showed a significant difference in grammaticality $(\mathrm{z}=4.5)$ such that the average grammaticality in the 2 -NP conditions was $98 \%$ compared to $85 \%$ in the experimental conditions. Recall that such a difference between 2-NP and 3-NP conditions was also found in the pilot study discussed earlier.

\begin{tabular}{|c|c|c|c|}
\hline \multicolumn{4}{|c|}{ Experiment 2a: Unique case-marker } \\
\hline & Coefficient & SE & Z-value \\
\hline Intercept & 1.71 & 0.34 & 4.9 \\
\hline ne-se-ko & 0.55 & 0.51 & 1.05 \\
\hline ko-ne-se & 0.72 & 0.54 & 1.33 \\
\hline ko-se-ne & 0.007 & 0.46 & 0.01 \\
\hline se-ko-ne & -0.27 & 0.44 & -0.61 \\
\hline se-ne-ko & -0.20 & 0.45 & -0.45 \\
\hline \multicolumn{4}{|c|}{ Experiment 2b: Similar case-marker } \\
\hline & Coefficient & SE & Z-value \\
\hline Intercept & 0.19 & 0.47 & 0.41 \\
\hline ko-se-ko & -2.84 & 0.50 & -5.63 \\
\hline se-ne-se & -0.85 & 0.40 & -2.12 \\
\hline se-ko-se & -3.14 & 0.52 & -5.99 \\
\hline \multicolumn{4}{|c|}{ Unique case-marker vs Similar case-marker } \\
\hline & Coefficient & $\mathrm{SE}$ & Z-value \\
\hline Intercept & 1.97 & 0.21 & 9.3 \\
\hline Similar case-marker & -2.79 & 0.21 & -13.22 \\
\hline
\end{tabular}

Table 3: glmer results for the grammaticality analysis for Experiment 2. All analysis uses treatment contrast. For the unique case-marker experiment, ne-ko-se was the baseline; for the similar case-marker experiment ko-ne-ko was the baseline. For the model comparing the two experiments, unique case-marker experiment was the baseline. $\mathrm{z}$-value more than 2 appear in bold text.

Table 4 shows the different types of errors for conditions with unique case-markers. One prominent trend was that the N2-N3 errors were much higher with N1-se conditions (i.e., se-ko-ne, se-ne-ko) compared to the rest of the conditions $(\mathrm{z}=3.5)$.

\subsubsection{Results: Similar case-marker (Experiment 2b)}

Results for the grammaticality analysis show that compared to the ko-ne-ko condition parsing errors were higher in other conditions (see, Table 3). The average parsing error due to incorrect prediction was around $67 \%$. This can be seen in Figure 5.

Table 4 shows different types of errors for the conditions with similar case-markers. The table shows that both N1-N2/N2-N3 errors as well as case-exchange errors were quite high

\footnotetext{
${ }^{9}$ There were around 7 such 2-NP filler items with differing case-markers (such as ko-se, ko-ne, ne-ko). In all there were 245 such data points.
} 

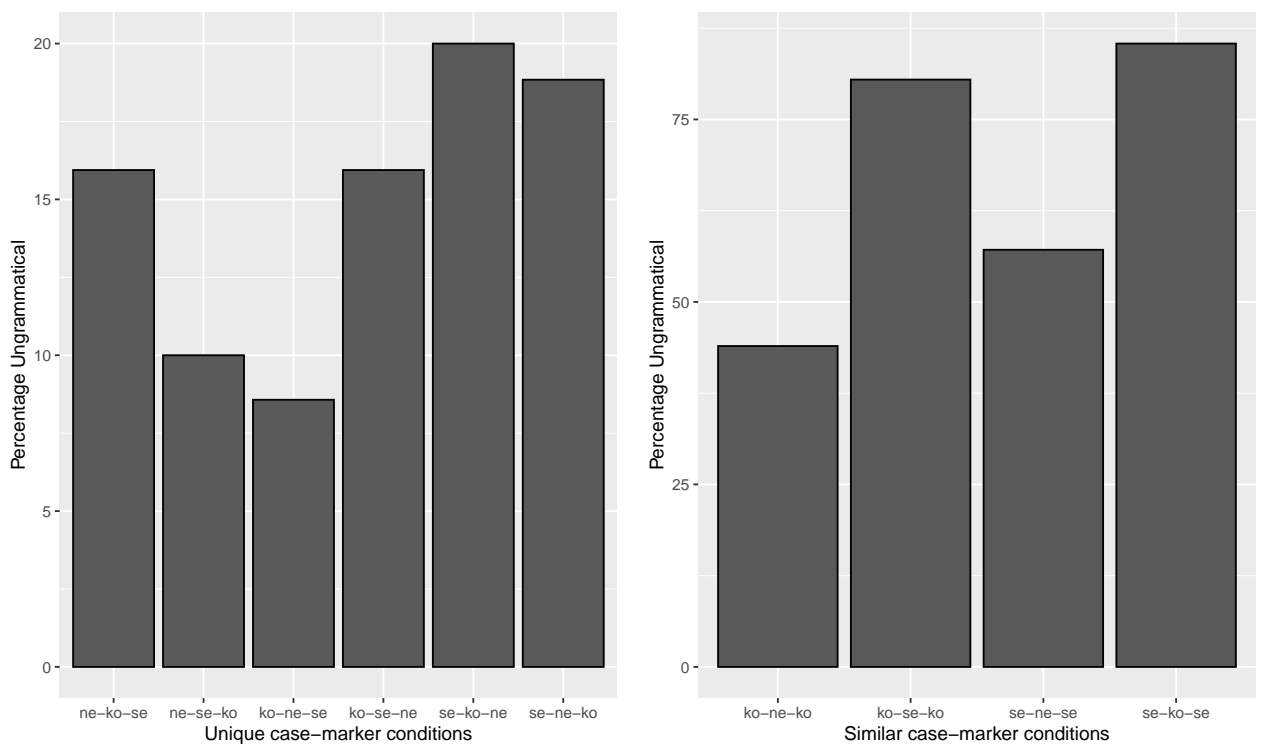

Figure 5: Percentage ungrammatical completions for all the conditions in the unique case-marker experiment (left) and the similar case-marker experiment (right). Percentage values were computed by considering the number of ungrammatical completions out of all the completions in a particular condition.

\begin{tabular}{|c|c|c|c|c|c|}
\hline \multicolumn{7}{|c|}{ Experiment 2a: Unique case-marker } \\
\hline Condition & N1-N2 & N2-N3 & N1-N3 & Case-exchange & Random \\
\hline ne-ko-se & 3 & 0 & 5 & 5 & 1 \\
\hline ne-se-ko & 3 & 1 & 4 & 0 & 0 \\
\hline ko-ne-se & 3 & 2 & 0 & 0 & 2 \\
\hline ko-se-ne & 1 & 6 & 5 & 1 & 1 \\
\hline se-ko-ne & 1 & 11 & 4 & 0 & 2 \\
\hline se-ne-ko & 0 & 12 & 6 & 0 & 1 \\
\hline \hline \multicolumn{7}{|c|}{ Experiment 2b: Similar case-marker } \\
\hline Condition & N1-N2/N2-N3 & N1-N3 & Case-exchange & Random \\
\hline ko-ne-ko & 23 & 0 & 7 & 10 \\
\hline ko-se-ko & 11 & 5 & 37 & 19 \\
\hline se-ne-se & 25 & 0 & 20 & 7 \\
\hline se-ko-se & 27 & 0 & 38 & 11 \\
\hline
\end{tabular}

Table 4: Parsing errors classification in Experiment 2a and Experiment 2b.

in this experiment. One prominent trend was that the case-exchange errors were much fewer in conditions where -ne appeared on N2 (i.e., ko-ne-ko, se-ne-se) compared to when it was not present $(\mathrm{z}=3.1)$. 


\subsubsection{Results: Comparision between unique case and similar case}

A comparison of ungrammatical completions in Experiment 2a (unique case-marker) with $2 \mathrm{~b}$ (similar case-marker) shows that errors in the latter were much higher than the former $(\mathrm{z}=-13.22)$; also see Figure 6 . Consistent with this trend both case-exchange as well as the $\mathrm{N} 2-\mathrm{N} 3$ errors were much higher in experiment $2 \mathrm{~b}$ compared to $2 \mathrm{a}(\mathrm{z}=2.89)$. In particular, while the number of case-exchange errors in experiment 2 a were quite less, such errors were much higher in experiment $2 \mathrm{~b}(\mathrm{z}=4.4)$.

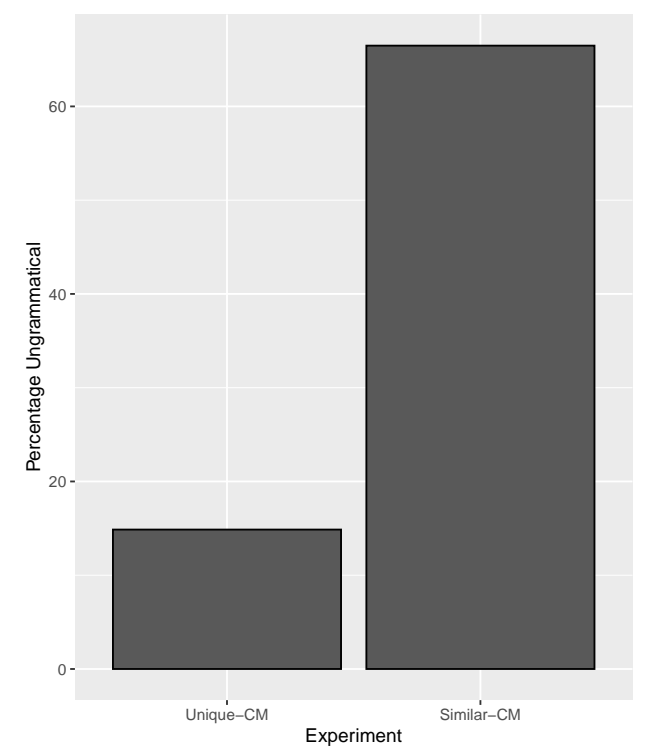

Figure 6: Percentage ungrammatical completions in the unique case-marker experiment (Unique-CM) vs similar case-marker experiment (Similar-CM).

\subsection{Discussion}

The results for the current experiments are consistent with the findings of the pilot study. Hindi native speakers make parsing errors due to incorrect predictions in various 3-NP conditions tested. The average error was found to be higher when the case-markers were repeated $(67 \%)$ compared to when the three case-markers were unique $(15 \%)$. In comparison, the average error in the 2-NP filler items was $2 \%$.

Together the two experiments highlight a limit on predictive processing in Hindi and therefore support the FPM hypothesis. The parser is susceptible to making verb prediction errors in 3-NP conditions with animate nouns. Results show that in such configurations, the parser tends to predict simple structures. Recall that the 3-NP conditions can be completed using a variety of syntactic configurations with differing complexity (operationalized as multiple verbs involving around 2-3 clausal embedding and around 5-9 syntactic relations; see, Table 2). Across the two experiments, close to $88 \%$ of grammatical completions had less than or equal to 2 clausal embedding and around $72 \%$ of grammatical completions had less than or equal to 5 verbal relations (see Table 5). Indeed, not a single instance of grammatical completion with a double embedded structure was found in the two experiments. Such 
a constraint on predictive processing could be due to the need to maintain multiple heads as well as multiple syntactic dependencies in the 3 -NP conditions (as seen in Table 2). It is reasonable to assume that positing such complex structure would increase the working memory load (cf. Gibson, 1998; Yngve, 1960; Lewis, 1993). On similar lines, the ungrammatical completions in the two experiments had simple structure. Table 6 shows that only $20 \%$ of all ungrammatical completions had 2 clauses and only $14 \%$ of all ungrammatical completions had more than 4 verbal relations in the posited structure.

\begin{tabular}{|c|c|c|c|c|}
\hline Completion & Count & Percentage & $\begin{array}{c}\text { No. of } \\
\text { clauses }\end{array}$ & $\begin{array}{c}\text { No. of core } \\
\text { relations }\end{array}$ \\
\hline T+DT & 170 & $35.78 \%$ & 2 & 5 \\
CAUS & 159 & $33.47 \%$ & 1 & 3 \\
N+DT+DT & 70 & $14.73 \%$ & 2 & 6 \\
T+T & 21 & $4.4 \%$ & 2 & 5 \\
others & 55 & $11.57 \%$ & - & - \\
\hline
\end{tabular}

Table 5: Grammatical completions across Experiment 2a and Experiment 2b.

\begin{tabular}{|c|c|c|c|c|}
\hline Completion & Count & Percentage & $\begin{array}{c}\text { No. of } \\
\text { clauses }\end{array}$ & $\begin{array}{c}\text { No. of core } \\
\text { relations }\end{array}$ \\
\hline CAUS & 50 & $16.6 \%$ & 1 & 3 \\
T+DT & 41 & $13.6 \%$ & 2 & 5 \\
T & 40 & $13.3 \%$ & 1 & 2 \\
N+DT & 34 & $11.3 \%$ & 1 & 3 \\
IN+DT & 18 & $6.0 \%$ & 2 & 4 \\
N+T & 16 & $5.3 \%$ & 1 & 3 \\
DT & 12 & $4.0 \%$ & 1 & 3 \\
N+CAUS & 10 & $3.3 \%$ & 1 & 3 \\
others & 89 & $26.3 \%$ & - & - \\
\hline
\end{tabular}

Table 6: Ungrammatical completions across Experiment 2a and Experiment 2b.

The results for the unique case-marker experiment found that the word order of the casemarker did not significantly affect the average number of errors in various 3-NP conditions. This suggests that the parser is generally vulnerable in such cases. At the same time, certain 3-NP conditions lead to specific kinds of errors, thus highlighting parsing strategy in these configurations. In particular, if the local case-marker combination is more frequent than the global case-marker combination, the local features tend to be used during the completion to form illicit parses that are globally inconsistent. A closer look at the nature of the error (see Table 4), shows that when the -se case-marker appears sentence initially, the number of N2-N3 errors were significantly higher compared to other conditions. For example, in the se-ne-ko condition, completions that are compatible with ne-ko become high. This could be 
because se-ne-ko is a very rare combination while ne-ko is quite frequent. ${ }^{10}$ Table 7 shows some examples of such errors.

On similar lines, in the case of similar case-marker conditions, if both global and local nominal features are rare, then the parser makes a prediction by misinterpreting one of the case-markers in order to make a (incorrect) verbal prediction. We call such parsing errors, case-exchange errors (see Table 4). For example, because the frequency of both se-ko-se and ko-se is less, the parser misinterprets the sentence fragment as ne-ko-se. ${ }^{11}$ Table 8 shows some examples of such errors. We discuss the implication of these errors in the unique and similar case-marker experiments on parsing strategy during comprehension in Section 6.

\begin{tabular}{|c|c|c|c|}
\hline Embeddings & Completion & Count & Example \\
\hline \multirow{4}{*}{1} & $\mathrm{~N}+\mathrm{DT}$ & 10 & Ntyt\$\& N1-ko N2-ne kuchh kaha 'something say' \\
\hline & $\mathrm{T}$ & 3 & N/t-\$\& N1-ko N2-ne peeta tha 'hit PAST' \\
\hline & CAUS & 1 & 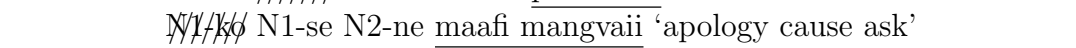 \\
\hline & DT & 1 & Ayty \$\& N1-ne N2-ko introduce kiya 'introduce do' \\
\hline \multirow{3}{*}{2} & $\mathrm{~N}+\mathrm{T}+\mathrm{DT}$ & 7 & Ayff \$p N1-ko N2-ne (prashna hal karne ko) kahaa 'question to solve ACC say' \\
\hline & $\mathrm{IN}+\mathrm{DT}$ & 6 & Xy1 $1 \$$ \& N1-ne N2-ko (chup rehne ko) kaha 'to stay quite say' \\
\hline & $\mathrm{N}+\mathrm{DT}+\mathrm{DT}$ & 1 & XY1 1 -s, N1-ne N2-ko (pen dene se) mana kar diya 'pen to give decline' \\
\hline
\end{tabular}

Table 7: Experiment 2a: N2+N3 errors in the unique case-marker experiment for conditions ko-se-ne, seko-ne, se-ne-ko. $v 1+v 2$ signifies an embedded structure with $v 1$ as the embedded non-finite verb and $v 2$ as the matrix verb. In the case of $n+v 1+v 2, n$ is part of the $v 1$ non-finite clause and v2 is the matrix verb. IN: Intransitive, CAUS: Causative, T: Transitive, DT: Ditransitive, N: Noun.

\begin{tabular}{|c|c|c|}
\hline Completion & Count & Example \\
\hline CAUS & 37 & N1-\$夕фne N2-ko N3-se milwaaya 'cause to meet' \\
\hline $\mathrm{T}+\mathrm{DT}$ & 35 & N1-k kq ne N2-se N3-ko milne ko kahaa 'meeting ACC ask' \\
\hline $\mathrm{N}+\mathrm{CAUS}$ & 7 & N1-kł $\phi$ ne N2-se N3-ko mithayi bhejwayi 'sweets cause to send' \\
\hline $\mathrm{N}+\mathrm{DT}+\mathrm{DT}$ & 7 & N1-\$ene N2-ko N3-se book lene ko kaha 'book to take ask' \\
\hline $\mathrm{DT}+\mathrm{DT}$ & 4 & N1-\$s dko N2-ne N3-se padhane ko kaha 'to study ask' \\
\hline $\mathrm{T}+\mathrm{T}$ & 4 & N1-\$, \$ko N2-ne N3-se milne k liye bulaya 'to meet invite' \\
\hline DT & 3 & N1-\$s \&ne N2-ko N3-se alag kar diya 'separate do' \\
\hline others & 5 & - \\
\hline
\end{tabular}

Table 8: Experiment 2b: Case-exchange errors in similar case-marker conditions. $v 1+v 2$ signifies an embedded structure with $v 1$ as the embedded non-finite verb and $v 2$ as the matrix verb. In the case of $n+v 1+v 2$, $n$ is part of the $v 1$ non-finite clause and $v 2$ is the matrix verb. IN: Intransitive, CAUS: Causative, T: Transitive, DT: Ditransitive, N: Noun.

To summarize, the nature of grammatical and ungrammatical completions show that Hindi native speakers tend to predict simple structures with fewer embeddings and minimal

\footnotetext{
${ }^{10}$ In a Hindi treebank Bhatt et al. (2009), there were 0 instance of se-ne-ko while there were 1231 instances of ne-ko.

${ }^{11}$ In a Hindi treebank Bhatt et al. (2009), there were 0 instance of se-ko-se, 79 instances of ko-se and 14 instances of ne-ko-se.
} 
number of core verbal relations. The $\mathrm{N} 2+\mathrm{N} 3 /$ case-exchange errors in the unique/similar case-marker experiments show a prediction strategy where the parser misinterprets rare input features (e.g., case-markers) as a frequent case-marker combination to form illicit parses at the expense of globally consistent parses. The results suggest that Hindi native speakers should find structures with more than 1 embeddings (i.e., 2 verbs) and more than 5 core verbal relations difficult to process. In addition, the fact that there were close to $7.7 \%$ instances where a 3-NP combination ( N1 N2 N3) was simplified as 2-NP coordination (i.e., $N 1 N_{2}$ and $\left.N_{3} N_{4}\right)$ to make verbal completions also reinforces this point.

The experiments discussed in this section highlighted prediction fallibility in Hindi. The nature of the parsing errors discussed above is not predicted by the RPM hypothesis. We next test another prediction of the RPM hypothesis, i.e., verbal predictions in SOV languages are robustly maintained in the face of additional preverbal elements (e.g., adjuncts).

\section{Experiment 3}

In experiment 3 we investigate the effect of preverbal adjuncts on verbal prediction and its maintenance. In particular, it tests a key claim of the RPM hypothesis that verbal predictions can be maintained robustly when additional linguistic material in the form of adjuncts is added preverbally (Levy, 2008; Vasishth \& Lewis, 2006; Vasishth et al., 2010).

\subsection{Material and methods}

\subsubsection{Participants}

27 native speakers of Hindi participated in this experiment. These subjects did not participate in any other experiment discussed in the paper. All the participants were undergraduate or graduate students from the Indian Institute of Technology, Delhi. The average age of the participants was $21.3(\mathrm{SD}=2.97)$. Each participant was paid INR 200 for participating in the experiment.

\subsubsection{Items}

We use the ne-ko-se condition from the previous experiment as the baseline condition. The other conditions added additional linguistic material of differing complexity after the 3rd noun, i.e., N3-se. This was done to investigate prediction maintenance while controlling for the strength of predictability at N3. The conditions can be seen in example 6 . In condition (b), two adverbials are introduced after N3. In condition (c), a non-finite clause (comprising of an intransitive verb and its Genetive marked subject) is introduced after N3. The adjuncts in conditions (b) and (c) can structurally attach only with the verbs predicted at N3. Additionally, the adjunct in condition (c) is assumed to be more complex than (b) - processing the adjunct in (c) requires creating an embedded structure while in (b) no such structure is formed. In conditions (d) and (e), a relative clause (RC) is added as an adjunct. The relative clause in these two conditions cannot structurally modify the upcoming verb and modifies N3 instead. Additionally, the RC in condition (e) is assumed to be more complex than the one in (d), as it involves a transitive event compared to the copular construction in (d). The adjuncts in conditions (b)-(e) comprise of 4-5 words. 
(6)
a. Baseline (ne-ko-se)
pUjaa-ne urmilaa-ko sunitaa-se ...
Pooja-ERG Urmila-ACC Sunita-ABL ...

b. Simple Verbal modification (Adverb)

pUjaa-ne urmilaa-ko sunitaa-se kal der shaam-me ...

Pooja-ERG Urmila-ACC Sunita-ABL yesterday late evening-LOC ...

c. Complex Verbal modification (Non-finite clause)

pUjaa-ne urmilaa-ko sunitaa-se police-ke jaane ke baad...

Pooja-ERG Urmila-ACC Sunita-ABL Police-GEN go-Part after ...

d. Simple Noun modification ( $\mathrm{RC}$ copula)

pUjaa-ne urmilaa-ko sunitaa-se jo dilli-me hai ...

Pooja-ERG Urmila-ACC Sunita-ABL who Delhi-LOC is ...

e. Complex Noun modification (RC transitive)

pUjaa-ne urmilaa-ko sunitaa-se jo hokI khelta hai ...

Pooja-ERG Urmila-ACC Sunita-ABL who hockey play PRES ...

25 sets of items were created with the above conditions. In addition, there were 50 filler items. A latin-square design was used to present the items. In all, 5 lists were prepared.

\subsubsection{Procedure}

Similar to experiment 1 , we follow the sentence completion paradigm in this experiment. See section 2.1.3 for details.

\subsection{Response coding}

The response coding procedure was the same as in experiment 2. See section 3.2 for details.

\subsection{Predictions}

The key assumption of the RPM hypothesis is that in SOV language predictions get better and are maintained robustly in the face of increased distance between the predicted verb and its preverbal modifiers (arguments or adjuncts). As stated earlier, this is how effects such as anti-locality and lack of structural forgetting is explained in the literature. Most experimental manipulations add such modifiers to demonstrate anti-locality in SOV languages such as German (Levy \& Keller, 2013; Konieczny, 2000), Hindi (Vasishth \& Lewis, 2006; Husain et al., 2014), Japanense (Nakatani \& Gibson, 2010). Theories that explain lack of forgetting effect in SOV languages (Vasishth et al., 2010) predict that addition of adjuncts (e.g., an embedded clause in conditions c, d, and e) will not affect the maintenance of the predicted verbs. This is because the processing system in SOV language becomes efficient in handling clause final verbs due to the high frequency of such SOV configurations in the language. Critically, the RPM hypothesis will not predict any degradation of verbal prediction when additional adjuncts (in the form of adverbial, participle, or a relative clause) are added after a robust (and grammatical) verbal prediction has been made at N3-se. 
The FPM hypothesis will predict that addition of adjuncts can degrade the prediction made at N3. In particular, the degradation in robust prediction should be higher in complex adjunct conditions such as participles and relative clauses.

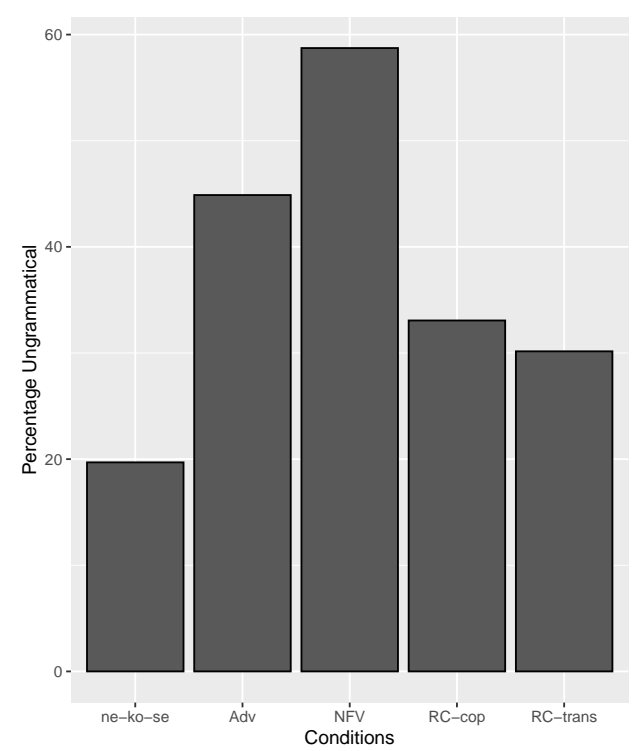

Figure 7: Percentage ungrammatical completions for all the conditions in Experiment 3.

\subsection{Results}

All statistical analyses have been done using the generalized linear mixed-effects model with logit link function. This has been done using the lme4 package (Bates et al., 2015) in R. Maximal models were fit when possible (Barr et al., 2013); in case of convergence failure, a less complex model was fit by successively removing the random slopes of the by-subject and by-item random effects component.

Figure 7 shows the percentage ungrammatical completions in all the conditions. ${ }^{12}$ The average ungrammatical completions in the baseline condition was $19.7 \%$, compared to that the average ungrammatical completions in the other conditions with adjuncts was $41.7 \%$. Table 9 shows the break-up for each condition. The results for grammaticality of the completions (grammatical vs ungrammatical) show that compared to the baseline condition (a), the percentage of grammatical completions was significantly lower in other conditions (b)-(d) (see Tables 10). ${ }^{13}$

Table 11 shows the parsing error types. We find that bulk of the errors in the nonbaseline conditions are due to N1-N2, N1-N3, and N1-N2/N1-N3, i.e., the completion is meaningful if one considers the nominal features of the first two nouns or the first and the

\footnotetext{
${ }^{12}$ The percentages consider the number of ungrammatical completions out of all the completions in a particular condition.

${ }^{13}$ Since the percentage errors in the two $\mathrm{RC}$ conditions were similar, they were collapsed for the purpose of the analysis. The reported results do not change if they are analyzed separately.
} 


\begin{tabular}{|c|c|c|c|}
\hline Condition & $\begin{array}{c}\text { Grammatical } \\
\text { completions }\end{array}$ & $\begin{array}{c}\text { Ungrammatical } \\
\text { completions }\end{array}$ & Total \\
\hline \hline ne-ko-se & $106(80.3 \%)$ & $26(19.6 \%)$ & 132 \\
\hline Adverbial & $70(55.1 \%)$ & $57(44.8 \%)$ & 127 \\
\hline Non-finite clause & $52(41.2 \%)$ & $74(58.7 \%)$ & 126 \\
\hline RC Copula & $83(66.9 \%)$ & $41(33.0 \%)$ & 124 \\
\hline RC Transitive & $88(69.8 \%)$ & $38(30.1 \%)$ & 126 \\
\hline
\end{tabular}

Table 9: Grammatical and ungrammatical completion counts in conditions a-e. Percentages shown in braces.

\begin{tabular}{|c|c|c|c|}
\hline & Coefficient & SE & z-value \\
\hline \hline Intercept & 1.63 & 0.29 & 5.52 \\
\hline Adverbial & -1.36 & 0.30 & $\mathbf{- 4 . 5 0}$ \\
\hline Non-finite clause & -2.04 & 0.31 & $\mathbf{- 6 . 6 0}$ \\
\hline RC & -0.69 & 0.27 & $\mathbf{- 2 . 5 5}$ \\
\hline
\end{tabular}

Table 10: glmer results for the grammatical vs ungrammatical completion. The analysis uses treatment contrast, with condition (a) as the baseline with other coefficient signifying the difference from the baseline.

\begin{tabular}{|c|c|c|c|c|c|c|}
\hline Condition & N1-N2 & N2-N3 & N1-N3 & N1-N2/N1-N3 & Case-exchange & Others \\
\hline ne-ko-se & 1 & 2 & 1 & 9 & 7 & 6 \\
\hline Adverbial & 14 & 3 & 5 & 23 & 3 & 8 \\
\hline Non-finite clause & 16 & 3 & 11 & 33 & 4 & 1 \\
\hline RC & 7 & 7 & 8 & 38 & 11 & 6 \\
\hline
\end{tabular}

Table 11: Parsing errors classification in Experiment 3.

last nouns. Compared to the baseline condition, all the other conditions had significantly more such errors $(\mathrm{z}=2.9, \mathrm{z}=4.2, \mathrm{z}=2.5$ respectively for conditions Adverb, Non-finite clause, $\mathrm{RC})$. Table 12 shows some prominent error completions based on the type of predicted verbs found across various conditions.

\begin{tabular}{|c|c|}
\hline Completion & Example \\
\hline$\overline{\mathrm{T}}$ & 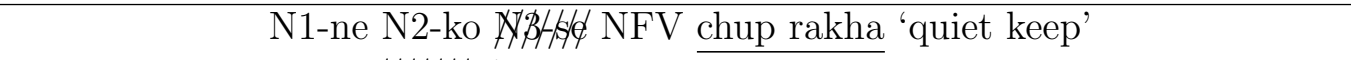 \\
\hline $\mathrm{IN}+\mathrm{DT}$ & N1-ne N2-ko X\$\$力 $\$$ ADV baag jaane ko kaha 'to run away ask' \\
\hline $\mathrm{N}+\mathrm{IN}+\mathrm{DT}$ & N1-ne N2-ko A \\
\hline $\mathrm{N}+\mathrm{T}+\mathrm{DT}$ & N1-ne A/4fk $\mathrm{N}$ 3-se RC kavita likhne ki maang ki 'poem to write request' \\
\hline
\end{tabular}

Table 12: Sample verb class errors in Experiment 3. $v 1+v 2$ signifies an embedded structure with $v 1$ as the embedded non-finite verb and $v 2$ as the matrix verb. In the case of $n+v 1+v 2, n$ is part of the $v 1$ non-finite clause and $v_{2} 2$ is the matrix verb. IN: Intransitive, CAUS: Causative, T: Transitive, DT: Ditransitive, N: Noun. 


\subsection{Discussion}

The results of the current experiment show that addition of adjuncts after the N1-ne N2ko N3-se string increases the percentage of ungrammatical completions. This goes counter to the robust prediction maintenance assumption of the RPM hypothesis and supports the FPM hypothesis.

The average correct prediction in the baseline condition was around $80 \%$, the result suggest that addition of adjuncts was detrimental to the maintenance of the predicted verbs in the baseline condition. This was particularly true when the adjunct was complex, e.g., a non-finite verb. This is not expected in an SOV language considering the proposals of linguistic adaptability that predict better maintenance of clause final verbal predictions (Vasishth et al., 2010; Levy \& Keller, 2013). However, under the assumption that the parser is susceptible to errors in 3-NP conditions (as noted in Experiment 2), presumably due to increased working memory load, addition of new linguistic material could lead to further increase in load and thereby lead to processing difficulty. This lends further support to the FPM hypothesis and highlights the configurations where parsing in SOV languages can suffer.

Qualitatively, the nature of predicted verb class in the grammatical and ungrammatical completions were similar to those found in Experiment 2. The dominant parsing error type observed in this experiment is also consistent with what was found in Experiment 2. In particular, when the parser is under strain due to the maintenance of various heads/relations, it can rely on more frequent nominal features that it has seen so far. For this experiment, this turns out to be N1-ne N2-ko or N1-ne N3-se both of which are much more frequent than the N1-ne N2-ko N3-se combination. ${ }^{14}$ Unlike the conditions in Experiment 2a/b, N2N3 errors or the case-exchange errors are fewer because the potential features do not form frequent patterns for the N1-ne N2-ko N3-se combination.

\section{Experiment 4}

Experiment 1-3 demonstrated that the parser is susceptible to making errors in certain syntactic configurations. We argued that these parsing errors happen because of the fallibility in verbal predictions due to working-memory load. In this experiment we use the sentence rating task to probe these error types further. The sentence rating paradigm has been effectively used to study parsing behaviour in a variety of experiments (e.g., Gibson \& Thomas, 1999; Vasishth et al., 2010; Lau \& Ferreira, 2005).

We report 2 sub-experiments to probe the ratings of errors in various unique case-marker conditions discussed in Section 2. Experiment 4a investigates errors in the canonical ne-ko-se condition while Experiment 4b investigates errors in the non-canonical se-ne-ko condition.

\footnotetext{
${ }^{14}$ In a Hindi treebank Bhatt et al. (2009), there were 13 instance of ne-ko-se while there were 1231 instances of ne-ko and 436 instances of ne-se.
} 


\subsection{Material and methods}

\subsubsection{Participants}

53 native speakers of Hindi participated in this experiment. These subjects did not participate in any other experiment discussed in the paper. All the participants were undergraduate or graduate students from the Indian Institute of Technology, Delhi. The average age of the participants was 23.5 ( $\mathrm{SD}=3.75)$. Each participant was paid INR 150 for participating in the experiment.

\subsubsection{Items}

We first discuss the items for the ne-ko-se experiment (Experiment 4a). Example 7 shows the sample items for this experiment. Example 7a is the grammatical condition, while Examples $7 \mathrm{~b}-\mathrm{d}$ are ungrammatical. Example $7 \mathrm{~b}$ can be deemed grammatical by misinterpreting aman-ko 'Aman-ACC' as aman- $\underline{k}$ 'Aman-GEN', hence it's a case-exchange error condition. On similar lines, Example 7c can be deemed grammatical by ignoring vaishali-ne 'vaishaliERG', hence it's a N2-N3 error condition. Finally, Example 7d is ungrammatical because it uses an intransitive verb which cannot be integrated in any way with the preceding nominals.

\section{a. Grammatical}

vaishali-ne aman-ko kabir-se paani dene ko kahaa

Vaishali-ERG Aman-ACC Kabir-ACC water give ACC asked

'Vaishali asked Kabir to give water to Aman.'

b. Ungrammatical (Case exchange)

* vaishali-ne aman-ko kabir-se baat karwaai

Vaishali-ERG Aman-ACC Kabir-ACC talk did

c. Ungrammatical (N2-N3)

* vaishali-ne aman-ko kabir-se kuch kaam hai

Vaishali-ERG Aman-ACC Kabir-ACC some work have

d. Ungrammatical (Intransitive)

* vaishali-ne aman-ko kabir-se rona hai

Vaishali-ERG Aman-ACC Kabir-ACC cry have

Experiment 4b comprised of the se-ne-ko conditions. Recall that the se-ne-ko condition has the highest N2-N3 error type in Experiment 2. Similar to Experiment 4a, this experiment has a Grammatical condition, an N2-N3 ungrammatical condition as well as an Intransitive ungrammatical condition. No case-exchange ungrammatical condition was investigated since the se-ne-ko condition in Experiment 2 did not elicit such an error type.

(8) a. Grammatical

kabir-se vaishali-ne aman-ko padhne-ko kahaa

Kabir-ABL Vaishali-ERG Aman-ACC study ask

'Vaishali asked Aman to study from Kabir.'

b. Ungrammatical (N2-N3) 


\title{
* kabir-se vaishali-ne aman-ko chup rahane-ko kahaa Kabir-ABL Vaishali-ERG Aman-ACC quite keep ask
}

\section{c. Ungrammatical (Intransitive)}

\author{
* kabir-se vaishali-ne aman-ko jaane lagaa \\ Kabir-ABL Vaishali-ERG Aman-ACC go put
}

For each experiment 16 sets of items were created. In addition there were 88 filler items. The filler items included a variety of construction types including items that looked similar to the critical items. Additionally, the filler items contained some very simple grammatical sentences and some outright ungrammatical sentences. As discussed later, these sentences were used to filter out non-attentive participants.

\subsubsection{Procedure}

Sentence rating task (Schütze \& Sprouse, 2014) was employed as the experimental paradigm. Each sentence was presented using the centered self-paced reading (SPR) paradigm. A similar paradigm was employed by Christiansen \& MacDonald (2009). Participants were provided a sentence and their task was to complete rate it on a Likert scale of 1 to 7 (1: Completely unacceptable; 7: Completely acceptable). Each sentence appeared on the screen in the self-paced reading format. Initially, a ' + ' sign appeared on the center of the computer screen. When the participant pressed the space-bar key this + sign got replaced with the first word of the sentence. Successive button presses displayed the remaining words of the sentence at the center of the screen. A space-bar press after the last region of a sentence made a text box appear on the screen. After typing the rating the participants pressed the 'enter' key to move to the next trial. The experiment was conducted using Douglas Rohde's Linger software ${ }^{15}$. Items were automatically randomized by Linger. As stated earlier, the sentence rating paradigm has been effectively used to study parsing behaviour in a variety of experiments (e.g., Gibson \& Thomas, 1999; Lau \& Ferreira, 2005; Frank \& Ernst, 2018; Tabor et al., 2004). These ratings have been found to correlate well with online tasks (e.g., Bailey, 2004; Tabor et al., 2004; Vasishth et al., 2010; Frank et al., 2016).

\subsection{Predictions}

If the completion errors found in Experiment $2 \mathrm{a}$ are due to certain parsing strategies and lead to grammatical illusions, then we expect the error conditions to be rated higher or similar to the grammatical condition. In particular, in Experiment 4a, the Ungrammatical case-exchange condition and the Ungrammatical N2-N3 condition should be rated lower than the Grammatical ne-ko-se condition. This is because, the ne-ko-se condition is not highly amenable either to the case-exchange error or the N2-N3 error. ${ }^{16}$ However, since there is a chance for such errors, these conditions should be rated higher than the Ungrammatical

\footnotetext{
${ }^{15}$ version 2.94

${ }^{16}$ The ko-se combination in a Hindi treebank is not very frequent; in addition, replacing either of the three case-markers in the ne-ko-se combination with some other case-marker does not lead to a more frequent combination relative to the ne-ko-se combination.
} 
Intransitive condition. On the other hand, in Experiment 4b, the Ungrammatical N2-N3 condition should be rated similar (or higher) to the Grammatical se-ne-ko condition. This is because the ne-ko combination in Hindi is relatively more frequent than the se-ne-ko combination. Finally, the N2-N3 condition in Experiment 4b should be rated higher than the Ungrammatical Intransitive condition.

\subsection{Results}

All statistical analyses was done using the linear mixed-effects model. This has been done using the lme4 package (Bates et al., 2015) in R. Maximal models were fit when possible (Barr et al., 2013); in case of convergence failure, a less complex model was fit by successively removing the random slopes of the by-subject and by-item random effects component. Rating data was scaled before analysis. The completely ungrammatical/grammatical filler items were used to exclude potential participants who were not performing the task as expected. We excluded all participants who had a mean rating of more than 4.6 for the ungrammatical fillers and a mean rating of less than 5.7 for the grammatical fillers. These thresholds were obtained by considering ratings above the 3rd quartile+ICQ and 1st quartile-ICQ respectively for the ungrammatical and grammatical ratings. This lead to the removal of 1 participant. The non-removal of these data points did not change the results reported for the two studies.
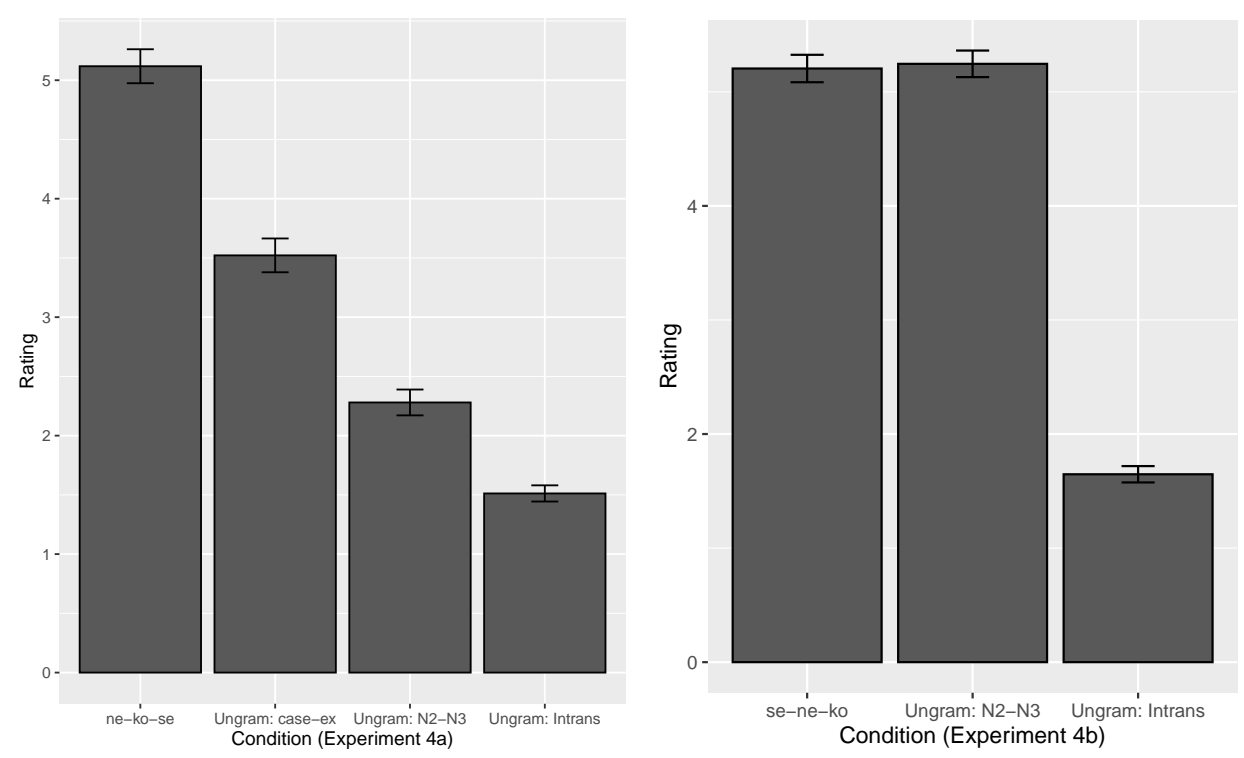

Figure 8: Average sentence ratings in all the conditions of Experiment 4a and Experiment $4 \mathrm{~b}$.

Figure 8 shows the mean ratings for all the conditions for Experiment 4a. Ratings were found to be in the following order - Grammatical ne-ko-se > Ungrammatical Case exchange $>$ Ungrammatical N2-N3 > Ungrammatical Intransitive. The mean ratings for these conditions were 5.1, 3.5, 2.3 and 1.5 respectively. Table 13 shows that the Grammatical ne-ko-se was significantly different from the other conditions. Changing the baseline condition to Ungrammatical Intransitive also resulted in a significant difference with other conditions. 


\begin{tabular}{|c|c|c|c|}
\hline \multicolumn{4}{|c|}{ Experiment 4a: ne-ko-se } \\
\hline & Coefficient & SE & t-value \\
\hline Intercept & 0.91 & 0.07 & 13.02 \\
\hline Ungram case-ex & -0.72 & 0.06 & -10.49 \\
\hline Ungram N2-N3 & -1.28 & 0.069 & -18.61 \\
\hline Ungram Intrans & -1.63 & 0.06 & -23.61 \\
\hline \multicolumn{4}{|c|}{ Experiment 4b: se-ne-ko } \\
\hline & Coefficient & $\mathrm{SE}$ & t-value \\
\hline Intercept & 0.49 & 0.10 & 4.71 \\
\hline Ungram N2-N3 & 0.008 & 0.13 & 0.06 \\
\hline Ungram Intrans & -1.47 & 0.10 & -14.01 \\
\hline
\end{tabular}

Table 13: lmer results for the rating in Experiment 4a and Experiment 4b. All analysis uses treatment contrast. For the Experiment 4a, ne-ko-se was the baseline; for Experiment 4b, se-ne-ko was the baseline.

Figure 8 also shows the mean ratings for all the conditions for Experiment 4b. Ratings were found to be in the following order - Grammatical se-ne-ko = Ungrammatical N2N3 > Ungrammatical Intransitive. The mean ratings for these conditions were 5.2, 5.2, and 1.6 respectively. Table 13 shows that the Grammatical se-ne-ko was not significantly different from the Ungrammatical N2-N3 condition, while it was significantly different from the Ungrammatical Intransitive condition.

\subsection{Discussion}

Results confirmed the prediction that Hindi native speakers are susceptible to grammatical illusion in certain word order configurations. In particular, ratings for the Ungrammatical N2-N3 in Experiment 4b were similar to the Grammatical se-ne-ko condition (cf. Gibson \& Thomas, 1999). This suggests that such ungrammatical completions found in Experiment 2 were due to certain parsing strategies that ignores the full preverbal features to form an illicit parse at the expense of a globally consistent parse.

The results also confirm the prediction that certain unlikely errors are rated low compared to the grammatical condition. The rating for Ungrammatical N2-N3 condition was much lower than the grammatical ne-ko-se rating. Recall that the ko-se combination is quite rare. This shows that errors of these kinds are less likely to go unnoticed by native speakers. At the same time, the ratings for the Ungrammatical case-exchange condition and Ungrammatical N2-N3 condition were significantly higher than the Ungrammatical Intransitive condition. This suggests that these condition could also lead to grammatical illusion. However, compared to the Ungrammatical N2-N3 condition in Experiment 4b this illusion might be less strong. Finally, a comparision of the baseline conditions in the two experiment with simple 2-NP fillers showed that the ne-ko-se condition was rated lower than the 2-NP filler items $(\mathrm{t}=-5.2)$ and the se-ne-ko condition was rated lower than the 2-NP filler items $(\mathrm{t}=-6.5)$. This is on expected lines and suggests that on average the participants find the 3 -NP items difficult to process. 
The results for Experiment 4b, therefore, provide evidence for grammatical illusion in an SOV language like Hindi. This is a novel finding considering the evidence for no-forgetting in other $\mathrm{SOV}^{17}$ languages such as German and Dutch (also see, Häussler \& Bader, 2015).

\section{General Discussion}

Experiments 1-4 show that Hindi native speakers successfully predict clause-final verbs based on the no. of preverbal nouns and their case-markers. At the same time, the results show verbal predictions in Hindi to be fallible. This fallibility is reflected as a significant amount of parsing errors in various experiments. In particular, the presence of 3 preverbal animate nouns with various case-markers lead to increased ungrammaticality. These errors further increased with the addition of preverbal adjuncts of differing complexity. A rating study probing various errors types demonstrated that some of the errors could be deemed as grammatical illusions, highlighting parser's vulnerability in such configurations. Together, the experiments demonstrate a limit to robust verbal prediction and its maintenance. The parsing process in an SOV language like Hindi seems to suffer in the face of establishing more than 5 verbal dependencies and more than 2 verbal heads (or more than 1 clausal embedding).

These results support the fallible prediction and maintenance (FPM) hypothesis that posits a limit to adaptability-based prediction in SOV languages. In particular, this hypothesis contends that increased working-memory load should affect the efficiency of the prediction system and lead to increased parsing errors. We could contrast this with the robust prediction and maintenance (RPM) hypothesis that posits an efficient adaptabilitybased prediction system in these languages. The RPM hypothesis has been used to account for effects such anti-locality, no-forgetting, etc. in SOV languages. The current work shows that the extent and scope of these effects needs a reappraisal. From a typological perspective, the results suggest that, similar to an SVO language like English, processing in an SOV language Hindi is affected by working-memory load due to syntactic configurations such as clausal embeddings.

\section{Predictive processing in SOV languages}

Understanding the nature of prediction is critical to our understanding of comprehension processes (Kuperberg \& Jaeger, 2016; Kuperberg, 2007; Huettig, 2015). While it is important to understand the output of the prediction system, it is equally important to understand how those predictions come about. What are the information sources and mechanisms used to make such predictions? Our work makes an important contribution to this line of work by highlighting the importance of investigating ungrammatical predictions. The nominal features that are used to make the ungrammatical predictions (see, Tables 7,8) provide some insight into the parsing process in these configurations. The error classification suggests that with increased complexity the parser uses those features that are found most frequently

\footnotetext{
${ }^{17}$ Technically, V2.
} 
in the language. This is done by selectively reconstructing the features of the previously seen context. Table 14 shows some cases of such frequently occurring reconstructions for various experimental configurations. For example, an N1-ne N2-ko N3-se NFV context is reconstructed as N1-ne N2-ko NFV by ignoring the features of one of the nouns (N3-se). Frequency counts for these configurations in a Hindi treebank suggests that the occurrence of N1-ne N2-ko is much higher than N1-ne N2-ko N3-se. Such a sensitivity to frequency of the context is also found in other examples shown in Table 14. A prediction system that assumes a reconstruction of its context during comprehension is broadly consistent with the proposal of noisy-channel model of language processing (e.g., Gibson et al., 2013; Kuperberg \& Jaeger, 2016; Piantadosi et al., 2011). Under such processing assumptions (cf. Kurumada \& Jaeger, 2015), the comprehension system is predictive due to the noisy transmission of information and unexpected input during comprehension can lead to effects such as local coherence (Tabor et al., 2004; Konieczny et al., 2009; Kamide \& Kukona, 2018), misinterpretation due to non-canonical order (Ferreira, 2003), etc.

\begin{tabular}{|c|c|c|c|c|}
\hline Original context & Frequency & & Reconstructed Context & Frequency \\
\hline N1-se N2-ne N3-ko & $3(.009 \%)$ & $\rightarrow$ & XN1-s@ N2-ne N3-ko & $1231(3.7 \%)$ \\
\hline N1-se N2-ko N3-ne. & $0(0 \%)$ & $\rightarrow$ & $X N 1-1 \$$ N 2 & $146(.44 \%)$ \\
\hline N1-se N2-ko N3-se & $0(0 \%)$ & $\rightarrow$ & N1-kidne N2-ko N3-se & $13(.03 \%)$ \\
\hline N1-ne N2-ko N3-se NFV & $0(0 \%)$ & $\rightarrow$ & 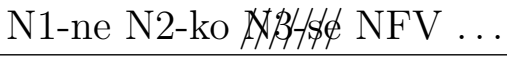 & $183(0.55 \%)$ \\
\hline
\end{tabular}

Table 14: Context reconstruction in some frequent errors for various experiments. NFV: Non-finite clause. Frequency counts computed from the Hindi dependency treebank (Bhatt et al., 2009). Percentages denote number of instances out of total instances of finite verbs in the treebank. Total number of finite verbs in the treebank $=32915$.

In particular, the contextual reconstruction examples shown in Table 14 seem to be compatible with the recently proposed lossy surprisal theory (Futrell \& Levy, 2017; Futrell et al., 2020) that assumes a noisy representation of previously seen input string. ${ }^{18}$ Such a noisy representation of the context is then used to make predictions and thereby such predictions could be ungrammatical. In other words, given a noisy representation, the context has to be reconstructed as faithfully as possible. The effectiveness of the reconstruction will depend on the frequency of the context in the language. If a context comprises of a frequent nominal pattern, its reconstruction will be better than unfamiliar patterns. Given that the N1-se N2-ne N3-ko configuration is rare in the language, its reconstruction could lead to a more frequent pattern of N2-ne N3-ko. The reconstructed context (N2-ne N3-ko) can then be used to make (ungrammatical) predictions. The increased errors in the similar case-marker conditions can also be explained using such an account. In particular, the frequency of similar case-marker combinations is relatively low than the frequency of unique case-marker combinations. A lossy surprisal account will therefore predict more reconstructions in the lower frequency similar case-marker conditions compared to the higher frequency

\footnotetext{
${ }^{18}$ In contrast, the surprisal metric (Hale, 2001; Levy, 2008) can only account for grammatical predictions. It has no provision for explaining systematic ungrammatical predictions such as the ones discussed in this work.
} 
unique case-marker conditions. Intrestingly, the N2-N3 errors are also compatible with the self-organizing parsing that assumes bottom-up information to generate locally consistent parses which then compete with globally consistent parses (Tabor et al., 2004). However, it is unclear how the errors caused due to the reconstruction of non-local context found in Experiment 3 (e.g., N1-ne N2-ko N3-se NFV $\rightarrow$ N1-ne N2-ko A 3 - \$ NFV) could be explained by a locally generated parse; here the context used for prediction is non-local.

The local as well as non-local errors could also be explained assuming a good-enough processing system (Ferreira et al., 2002). Similar to the noisy-channel model, the good-enough hypothesis also assumes that speakers make mistakes and therefore comprehenders rely on heuristics to accommodate such input to arrive at meaning. Indeed, the lossy-surprisal metric could be understood as an implementation of the good-enough comprehension system (Futrell et al., 2015). Under a good-enough system, the parser can rely on very little information from the input, leading to a shallow or incorrect interpretation of the received input. It has been proposed that a processing of such a kind could be a result of limits on time and resources that the parsing system is typically subject to (Ferreira \& Patson, 2007). From this perspective, the fallible prediction and maintenance hypothesis can be understood as a special case of the good-enough parsing hypothesis, where the parsing system relies on selective information for prediction when there is increased processing load. Such a prediction mechanism has also been demonstrated in English using a visual-world paradigm where local-coherence was shown to guide anticipation (Kamide \& Kukona, 2018). The current work therefore illustrates the overarching preference of the parser to posit simple structures (cf. Ferreira \& Patson, 2007; Frazier, 1985; Frazier \& Fodor, 1978; Fodor \& Inoue, 2000; Husain \& Yadav, 2020; Gibson \& Thomas, 1999) and highlights some factors under which good-enough processing might happen in an SOV language.

\section{Processing implication of prediction fallibility in SOV languages}

The completion study shows that with 3 preverbal animate nouns only 2 verb phrases are predicted (i.e., 1 clasual embedding). Indeed, the rating study discussed in Section 5 suggests that with certain 3-NP conditions, grammatical illusion can be induced with just 1 clausal embedding. This implies that in these configurations, the no-forgetting effects with 2 clausal embeddings found in German and Dutch should be difficult. Relatedly, a recent study investigating V2 forgetting effects in Hindi (Husain \& Bhatia, 2018) finds that experimental manipulations in such experiments should not just investigate the number of verbs, but also the number of the verbal dependencies. They find that a configuration such as (N1 N2 (N3 (IN.NFV) DT.NFV) DT) would be less susceptible to the forgetting effect than (N1 N2 (N3 (N4 DT.NFV) DT.NFV) DT). Table 2 shows that while both these configurations have 3 clauses, the former has 7 dependencies to establish while the latter has 9 dependencies to establish. This suggests that the no-forgetting effects in SOV languages should be probed further to probe the nature of dependencies formed in the configurations tested in such experiments.

Note that the claim is not that there is no prediction during comprehension in SOV lan- 
guages. Native speakers do get surprised when expectations are not met during comprehension (e.g., Boston et al., 2008; Husain et al., 2014; Agrawal et al., 2017) and comprehension is known to be driven by top-down processing (Kuperberg, 2007; Kuperberg \& Jaeger, 2016). The current set of experiments do demonstrate a robust prediction mechanism in a variety of syntactic configuration. In particular, prediction is robust when syntactic configurations are simple and frequent (e.g., in the 1-NP and 2-NP conditions). However, given the assumed central role of prediction in SOV languages, it becomes important to understand its scope and limit. Our work suggests that there are limits to verbal prediction in an SOV language like Hindi and highlights the need for a closer scrutiny of certain effects known in the literature that are driven by robust predictive processing in such languages. For example, in a recent study, Apurva \& Husain (2020) show that, while the completion study does not show any difference in verb prediction for short vs long noun-verb distance conditions, the SPR study shows a faster RT at the critical verb with increased noun-verb distance compared to short distance. They find such an effect in multiple studies and suggest that anti-locality effect in Hindi could be a results of the parser's expectation of seeing the location of the clause final verb rather than signifying robust prediction of a particular verb (class) and its maintenance. In effect, the reported effect could be due to a shallow parsing strategy (Ferreira et al., 2002; Ferreira \& Patson, 2007).

In the light of prediction fallibility during parsing SOV languages, it is interesting to note that previous research on processing of word-order variation and influence of case-marker in Hindi (and indeed, other SOV languages) has primarily focused on retrieval processes at the critical verb (e.g., Vasishth, 2003; Häussler \& Bader, 2015; Mishra et al., 2011; Lewis \& Nakayama, 2002). This line of work has investigated the influence of linear distance and similarity-based interference on the retrieval of arguments at the verb. However, given the current results that highlight the fallibility of prediction in Hindi, it is unclear what the processing cost at the verb signifies in such previous studies. Future research on SOV languages should therefore tease apart the interaction of predictive and integration processes during processing (cf. Vasishth \& Drenhaus, 2011; Husain et al., 2014; Ferreira \& Chantavarin, 2018).

\section{Working-memory constraints and SOV languages}

A key contribution of this work is to also highlight the role of working-memory constraint on predictive processing. For example, the conditions with 3 preverbal nouns lead to more parsing errors compared to the conditions with 2 preverbal nouns. One could argue that the errors in 2-NP conditions are less due to their high frequency in the language compared to the 3-NP conditions. However, a working-memory account need not be mutually exclusive to the frequency account. For example, processing difficulty during comprehension due to low frequency can be due to working-memory constraints during production (e.g., MacDonald, 2013; Kurumada \& Jaeger, 2015; Scontras et al., 2015). In addition, the differential parsing error due to intervener complexity discussed in Experiment 3 cannot be easily explained by a purely frequency based account. This is because all the intervener conditions in Experiment 3 are rare, however errors are high when the intervener is more complex vs when it's simple. 
Indeed, the current proposal of lossy surprisal (Futrell et al., 2020) that assumes a random noise function will also be unable to correctly account for this differential prediction error. If the linguistic material of adjuncts that follow the preverbal nominals have equal length, then a random noise function will not be able to capture differential prediction patterns due to the nature of adjuncts. Finally, the nature of parsing error during the completion task also highlights the influence of limited memory resource on parsing. As noted earlier, in the 3-NP conditions, the parser posits structures that have fewer verbal heads and have fewer dependency relations. Tables 5, 6 show that the number of clauses in such predictions range from 1-2, while the number of core syntactic relations range from 3-6. This can be contrasted with Table 2 which shows that range of clauses and core dependency relations for grammatical completions in 3-NP conditions can range from 1-3 and 3-9 respectively. A comparison of the complexity of parses in grammatical vs ungrammatical completion suggests that both the number of clauses as well as the number of core syntactic relations matter during the prediction process. The errors show that predicting/maintaining more than 2 verbs/clauses is rare. And in cases where the number of predicted verbs is similar between grammatical and ungrammatical completions, the total number of core relations in error completions is comparatively less.

Predictive processing in SOV languages has been frequently pitted against locality constraints because effects such as anti-locality and lack of forgetting go against workingmemory constraints during comprehension. SOV languages have been shown to have longer dependency length wrt SVO languages (Futrell et al., 2015; Yadav et al., 2020). Thus, the parser in SOV languages has been argued to be deeply influenced by certain typological properties such as the word order (Vasishth et al., 2010; Levy \& Keller, 2013). While this may be true to some extent, more recent corpus studies have also highlighted constraints on this adaptability. For example, using a cross-linguistic corpus study, Yadav et al. (2020) have shown that SOV languages do not allow for more than 3 clausal embeddings. Relatedly, Sharma et al. (2020) show that the core verbal arguments (subject, object, indirect objects) are typically found close to the verbal head and bulk of long distance dependencies in a language like Hindi are due verbal adjuncts. Indeed, the work suggests that processing in SOV languages should be susceptible to center-embedding similar to that in SVO languages.

Indeed, avoidance of complex preverbal configurations due to working memory constraints has been argued to be an important feature in SOV languages (e.g., Gibson et al., 2013; Ueno \& Polinsky, 2009; Ros et al., 2015). Consistent with these observations, the configurations used in the current study show that when working-memory is strained, the robustness of verbal prediction decreases. The current work shows that, in an SOV language like Hindi, the adaptability of the parser to make verbal predictions and maintain them has limits.

\section{Conclusion}

Given the importance of predictive processing in SOV languages, the current study highlights a limit to prediction in an SOV language like Hindi. Using a sentence completion task, we show that Hindi native speakers make consistent parsing errors in conditions with 3 ani- 
mate nouns with unique/similar case-markers. Such parsing errors increase when adjuncts of differing complexity follow the 3 nouns. The grammatical and ungrammatical completions show that native speakers of Hindi predict simple structures with at most 2 embedding and 5 core syntactic relations, thus highlighting an upper bound to robust verbal prediction and its maintenance in such configurations. A rating study showed that certain errors in such configurations can lead to grammatical illusion. Further, the type of parsing errors suggests that the parser relies on frequent preverbal nominal features in order to make verbal predictions and form illicit parses at the expense of globally consistent parses. Such a strategy can lead to a selective reconstruction of certain preverbal features and is compatible with a noisy channel model of comprehension. Together, the experiments paint a more nuanced picture of predictive processing in an SOV language like Hindi in terms of nature of verbal predictions and the possible cause of such predictions. The results highlight the need for a reappraisal of current theories that use robust prediction in SOV languages as an explanation to effects such as anti-locality and lack of forgetting.

\section{Acknowledgment}

This work was supported by a Department of Science and Technology - Cognitive Science Research Initiative grant (SR/CSRI/29/2015) to Samar Husain.

\section{References}

Agrawal, A., Agarwal, S., \& Husain, S. (2017). Role of expectation and working memory constraints in hindi comprehension: An eyetracking corpus analysis. Journal of Eye Movement Research, 10, 1-15.

Altmann, G. T., \& Kamide, Y. (1999). Incremental interpretation at verbs: Restricting the domain of subsequent reference. Cognition, 73, 247-256.

Altmann, G. T., \& Kamide, Y. (2007). The real-time mediation of visual attention by language and world knowledge: Linking anticipatory (and other) eye movements to linguistic processing. Journal of Memory and Language, 57, 502-518.

Apurva, \& Husain, S. (2020). Anti-locality effect in hindi: Evidence for shallow parsing. In submission, .

Ashby, J., Rayner, K., \& Charles Clifton, J. (2005). Eye movements of highly skilled and average readers: Differential effects of frequency and predictability. The Quarterly Journal of Experimental Psychology Section A, 58, 1065-1086.

Bailey, K. G. B. (2004). Disfluent speech and the visual world: An application of the visual world paradigm to the study of spoken language comprehension. Michigan State University, East Lansing, MI.. Ph.D. thesis Unpublished Doctoral Dissertation.

Barr, D. J., Levy, R., Scheepers, C., \& Tily, H. J. (2013). Random effects structure for confirmatory hypothesis testing: Keep it maximal. Journal of memory and language, 68, 255-278.

Bates, D., Machler, M., Bolker, B., \& Walker, S. (2015). Fitting linear mixed-effects models using lme4. Journal of Statistical Software, 67, 1-48.

Bhatia, S., \& Husain, S. (2018). Forgetting effects due to local coherence in hindi. In 31st Annual CUNY Sentence Processing Conference. UC Davis USA.

Bhatia, S., \& Husain, S. (2020). Preverbal syntactic complexity leads to local coherence effects. In submission, .

Bhatt, R., Narasimhan, B., Palmer, M., Rambow, O., Sharma, D. M., \& Xia, F. (2009). A multirepresentational and multi-layered treebank for Hindi/Urdu. In Proceedings of the Third Linguistic Annotation Workshop (pp. 186-189). Association for Computational Linguistics. 
Boston, M., Hale, J., Kliegl, R., Patil, U., \& Vasishth, S. (2008). Parsing costs as predictors of reading difficulty: An evaluation using the potsdam sentence corpus. Journal of Eye Movement Research), 2, $1-12$.

Boston, M. F., Hale, J. T., Vasishth, S., \& Kliegl, R. (2011). Parallel processing and sentence comprehension difficulty. Language and Cognitive Processes, 26, 301-349.

Christiansen, M. H., \& MacDonald, M. C. (2009). A usage-based approach to recursion in sentence processing. Language Learning, 59, 126-161.

DeLong, K. A., Troyer, M., \& Kutas, M. (2014). Pre-processing in sentence comprehension: Sensitivity to likely upcoming meaning and structure. Language and Linguistics Compass, 8, 631.

Dikker, S., Rabagliati, H., Farmer, T. A., \& Pylkkänen, L. (2010). Early occipital sensitivity to syntactic category is based on form typicality. Psychological Science, 21, 629-634.

Dikker, S., Rabagliati, H., \& Pylkkänen, L. (2009). Sensitivity to syntax in visual cortex. Cognition, 110, 293-321.

Farmer, T. A., Brown, M., \& Tanenhaus, M. K. (2013). Prediction, explanation, and the role of generative models in language processing. Behavioral and Brain Sciences, 36, 211.

Ferreira, F. (2003). The misinterpretation of noncanonical sentences. Cognitive Psychology, 47, $164-203$.

Ferreira, F., Bailey, K. G., \& Ferraro, V. (2002). Good-enough representations in language comprehension. Current Directions in Psychological Science, 11, 11-15.

Ferreira, F., \& Chantavarin, S. (2018). Integration and prediction in language processing: A synthesis of old and new. Current Directions in Psychological Science, 27, 443-448.

Ferreira, F., \& Patson, N. D. (2007). The 'good enough' approach to language comprehension. Language and Linguistics Compass, 1, 71-83.

Fischler, I. S., \& Bloom, P. A. (1979). Automatic and attentional processes in the effects of sentence contexts on word recognition. Journal of Verbal Learning and Verbal Behavior, 18, 1-20.

Fodor, J. D., \& Inoue, A. (2000). Garden path reanalysis: attach (anyway) and revision as last resort. In M. DiVincenzi, \& V. Lombardo (Eds.), Cross-Linguistic Perspectives in Language Processing (pp. 21-61). Dordrecht, The Netherlands: Kluwer.

Frank, S. L., \& Ernst, P. (2018). Judgements about double-embedded relative clauses differ between languages. Psychological Research, .

Frank, S. L., Trompenaars, T., \& Vasishth, S. (2016). Cross-linguistic differences in processing doubleembedded relative clauses: Working-memory constraints or language statistics? Cognitive Science, 40, $554-578$.

Frazier, L. (1985). Syntactic complexity. In L. K. D. Dowty, \& A. Zwicky (Eds.), Natural Language Parsing (pp. 129-189). Cambridge: Cambridge University Press.

Frazier, L., \& Fodor, J. D. (1978). The sausage machine: A new two-stage parsing model. Cognition, 6, 291-325.

Friederici, A. D., \& Frisch, S. (2000). Verb argument structure processing: The role of verb- specific and argument-specific information. Journal of Memory and Language, 43, 476-507.

Futrell, R., Gibson, E., \& Levy, R. (2020). Lossy-context surprisal: An information-theoretic model of memory effects in sentence processing, . Cognitive Science.

Futrell, R., \& Levy, R. (2017). Noisy-context surprisal as a human sentence processing cost model. In Proceedings of the 15th Conference of the European Chapter of the Association for Computational Linguistics (EACL) (p. 688-698).

Futrell, R., Mahowald, K., \& Gibson, E. (2015). Large-scale evidence of dependency length minimization in 37 languages. Proceedings of the National Academy of Sciences, 112, 10336-10341.

Gibson, E. (1998). Linguistic complexity: Locality of syntactic dependencies. Cognition, 68, 1-76.

Gibson, E., Piantadosi, S. T., Brink, K., Bergen, L., Lim, E., \& Saxe, R. (2013). A noisy-channel account of crosslinguistic word-order variation. Psychological Science, 24, 1079-1088.

Gibson, E., \& Thomas, J. (1999). Memory limitations and structural forgetting: The perception of complex ungrammatical sentences as grammatical. Language and Cognitive Processes, 14(3), 225-248.

Grodner, D., \& Gibson, E. (2005). Consequences of the serial nature of linguistic input for sentenial 
complexity. Cognitive Science, 29, 261-290.

Hale, J. (2001). A probabilistic earley parser as a psycholinguistic model. In Proceedings of the second meeting of the North American Chapter of the Association for Computational Linguistics on Language technologies (pp. 1-8). Association for Computational Linguistics.

Hale, J. T. (2006). Uncertainty about the rest of the sentence. Cognitive Science, 30.

Harinuma, S. (1999). Syntactic difficulty in english and japanese: a textual study. UCL Working Papers in Linguistics, 11, 309-322.

Hawkins, J. A. (1994). A performance theory of order and constituency volume 73. Cambridge University Press.

Hawkins, J. A. (2014). Cross-linguistic variation and efficiency. Oxford.

Huettig, F. (2015). Four central questions about prediction in language processing. Brain Research, 1626, $118-135$.

Husain, S., \& Bhatia, S. (2018). Processing hindi doubly center-embedded structures. In Proceedings of the 5th Annual Conference of The Association of Cognitive Science. Guwahati, India.

Husain, S., Vasishth, S., \& Srinivasan, N. (2014). Strong Expectations Cancel Locality Effects: Evidence From Hindi. PloS one, 9, e100986.

Husain, S., Vasishth, S., \& Srinivasan, N. (2015). Integration and prediction difficulty in Hindi sentence comprehension: Evidence from an eye-tracking corpus. Journal of Eye Movement Research, 8(2), 1-12.

Husain, S., \& Yadav, H. (2020). Target complexity modulates syntactic priming during comprehension. Frontiers in Psychology, 11, 454.

Häussler, J., \& Bader, M. (2015). An interference account of the missing-vp effect. Frontiers in Psychology, 6,766 .

Jäger, L., Chen, Z., Li, Q., Lin, C.-J. C., \& Vasishth, S. (2015). The subject-relative advantage in chinese: Evidence for expectation-based processing. Journal of Memory and Language, 79, 97-120.

Kachru, Y. (2006). Hindi. John Benjamins Publishing Company, Philadelphia.

Kamide, Y., Altmann, G. T., \& Haywood, S. L. (2003). The time-course of prediction in incremental sentence processing: Evidence from anticipatory eye movements. Journal of Memory and Language, 49, $133-156$.

Kamide, Y., \& Kukona, A. (2018). The influence of globally ungrammatical local syntactic constraints on real-time sentence comprehension: Evidence from the visual world paradigm and reading. Cognitive Science, 42, 2976-2998.

Kimball, J. (1973). Seven principles of surface structure parsing in natural language. Cognition, 2, 15 - 47.

Kliegl, R., Grabner, E., Rolfs, M., \& Engbert, R. (2004). Length, frequency, and predictability effects of words on eye movements in reading. European Journal of Cognitive Psychology, 16, 262-284.

Kliegl, R., Nuthmann, A., \& Engbert, R. (2006). Tracking the mind during reading: The influence of past, present, and future words on fixation durations. Journal of Experimental Psychology: General, 135, $12-35$.

Konieczny, L. (2000). Locality and parsing complexity. Journal of Psycholinguistic Research, 29, 627-645.

Konieczny, L., Müller-Feldmeth, D., Hachmann, W., Schwarzkopf, S., \& Wolfer, S. (2009). Local syntactic coherence interpretation. evidence from a visual world study. In Proceedings of the 31th Annual Conference of the Cognitive Science Society (pp. 1133-1138). Austin, TX: Cognitive Science Society.

Koso, A., Ojima, S., \& Hagiwara, H. (2011). An event-related potential investigation of lexical pitch-accent processing in auditory japanese. Brain research, 1385, 217-228.

Kuperberg, G. R. (2007). Neural mechanisms of language comprehension: Challenges to syntax. Brain Research, 1146, 23-49.

Kuperberg, G. R., \& Jaeger, T. F. (2016). What do we mean by prediction in language comprehension? Language, Cognition and Neuroscience, 31, 32-59.

Kurumada, C., \& Jaeger, T. F. (2015). Communicative efficiency in language production: Optional casemarking in japanese. Journal of Memory and Language, 83, $152-178$.

Kutas, M., DeLong, K. A., \& Smith, N. J. (2011). A look around at what lies ahead: Prediction and predictability in language processing. In M. Bar (Ed.), Predictions in the brain: Using our past to 
generate a future (pp. 190-207). New York, NY: Oxford University Press.

Kutas, M., \& Hillyard, S. (1984). Brain potentials during reading reflect word expectancy and semantic association. Nature, 307, $161-163$.

Kutas, M., \& Hillyard, S. A. (1980). Reading senseless sentences: Brain potentials reflect semantic incongruity. Science, 20\%, 203-205.

Lau, E. F., \& Ferreira, F. (2005). Lingering effects of disfluent material on comprehension of garden path sentences. Language and Cognitive Processes, 20, 633-666.

Levy, R. (2008). Expectation-based syntactic comprehension. Cognition, 106, 1126-1177.

Levy, R., \& Keller, F. (2013). Expectation and locality effects in german verb-final structures. Journal of memory and language, 68, 199-222.

Lewis, R. (1993). An Architecturally-based Theory of Human Sentence Processing. PhD Thesis, Carnegie Mellon University Pittsburgh, PA.. Ph.D. thesis Ph.D. dissertation.

Lewis, R. L., \& Nakayama, M. (2002). Syntactic and positional similarity effects in the processing of japanese embeddings. Sentence processing in East Asian languages, (pp. 85-110).

Lewis, R. L., \& Vasishth, S. (2005). An activation-based model of sentence processing as skilled memory retrieval. Cognitive science, 29, 375-419.

Lowder, M. W., Choi, W., Ferreira, F., \& Henderson, J. M. (2018). Lexical predictability during natural reading: Effects of surprisal and entropy reduction. Cognitive Science, 42, 1166-1183.

Luke, S. G., \& Christianson, K. (2015). Predicting inflectional morphology from context. Language, Cognition and Neuroscience, 30, 735-748.

Luke, S. G., \& Christianson, K. (2016). Limits on lexical prediction during reading. Cognitive Psychology, $88,22-60$.

MacDonald, M. C. (2013). How language production shapes language form and comprehension. Frontiers in Psychology, 4, 226.

Marslen-Wilson, W. (1973). Linguistic structure and speech shadowing at very short latencies. Nature, 244, $522-523$.

McRae, K., Spivey-Knowlton, M. J., \& Tanenhaus, M. K. (1998). Modeling the influence of thematic fit (and other constraints) in on-line sentence comprehension. Journal of Memory and Language, 38, 283312 .

Miller, G. A., \& Chomsky, N. (1963). Finitary models of language users. In R. B. R.D. Luce, \& E. Galanter (Eds.), Handbook of Mathematical Psychology (pp. 419-492). New York: Wiley volume 2.

Mishra, R. K., Pandey, A., \& Srinivasan, N. (2011). Revisiting the scrambling complexity hypothesis in sentence processing: a self-paced reading study on anomaly detection and scrambling in hindi. Reading and Writing, 24, 709-727.

Nakatani, K., \& Gibson, E. (2010). An on-line study of japanese nesting complexity. Cognitive Science, 34 , 94-112.

Petten, C. V., \& Luka, B. J. (2012). Prediction during language comprehension: Benefits, costs, and erp components. International Journal of Psychophysiology, 83, 176-190.

Piantadosi, S. T., Tily, H., \& Gibson, E. (2011). Word lengths are optimized for efficient communication. Proceedings of the National Academy of Sciences, 108, 3526-3529.

Pickering, M. J., \& Gambi, C. (2018). Predicting while comprehending language: A theory and review. Psychological Bulletin, 144, 1002-1044.

Pickering, M. J., \& Garrod, S. (2013). An integrated theory of language production and comprehension. Behavioral and Brain Sciences, 36, 329-347.

Rayner, K., Slattery, T. J., Drieghe, D., \& Liversedge, S. P. (2011). Eye movements and word skipping during reading:. Journal of Experimental Psychology: Human Perception and Performance, 37, 514 528 .

Ros, I., Santesteban, M., Fukumura, K., \& Laka, I. (2015). Aiming at shorter dependencies: the role of agreement morphology. Language, Cognition and Neuroscience, 30, 1156-1174.

Schütze, C. T., \& Sprouse, J. (2014). Experimental paradigms in psycholinguistics. In R. J. Podesva, \& D. Sharma (Eds.), Research Methods in Linguistics (pp. 27-50). Cambridge University Press. 
Schwanenflugel, P. J., \& Lacount, K. L. (1988). Semantic relatedness and the scope of facilitation for upcoming words in sentences. Journal of Experimental Psychology: Learning Memory and Cognition, 14, 344-354.

Schwanenflugel, P. J., \& Shoben, E. J. (1985). The influence of sentence constraint on the scope of facilitation for upcoming words. Journal of Memory and Language, 24, 232-252.

Scontras, G., Badecker, W., Shank, L., Lim, E., \& Fedorenko, E. (2015). Syntactic complexity effects in sentence production. Cognitive Science, 39, 559-583.

Sharma, K., Futrell, R., \& Husain, S. (2020). What determines the order of verbal dependents in hindi? effects of efficiency in comprehension and production. In Proceedings of Cognitive Modeling and Computational Linguistics (CMCL).

Staub, A. (2015). The effect of lexical predictability on eye movements in reading: Critical review and theoretical interpretation. Language and Linguistics Compass, 9, 311-327.

Staub, A., \& Clifton, J., C. (2006). Syntactic prediction in language comprehension: Evidence from either ... or. Journal of Experimental Psychology: Learning, Memory, and Cognition, 32, 425-436.

Staub, A., Grant, M., Astheimer, L., \& Cohen, A. (2015). The influence of cloze probability and item constraint on cloze task response time. Journal of Memory and Language, 82, 1-17.

Tabor, W., Galantucci, B., \& Richardson, D. (2004). Effects of merely local syntactic coherence on sentence processing. Journal of Memory and Language, 50, 355 - 370.

Taylor, W. (1953). 'cloze' procedure: A new tool for measuring readability. Journalism Quarterly, 30, 415-433.

Ueno, M., \& Polinsky, M. (2009). Does headedness affect processing? a new look at the vo-ov contrast. Journal of Linguistics, 45, 675-710.

Vasishth, S. (2003). Working memory in sentence comprehension: Processing Hindi center embeddings. Routledge.

Vasishth, S., \& Drenhaus, H. (2011). Locality in German. Dialogue \& Discourse, 2, 59-82.

Vasishth, S., \& Lewis, R. L. (2006). Argument-head distance and processing complexity: Explaining both locality and antilocality effects. Language, (pp. 767-794).

Vasishth, S., Suckow, K., Lewis, R. L., \& Kern, S. (2010). Short-term forgetting in sentence comprehension: Crosslinguistic evidence from verb-final structures. Language and Cognitive Processes, 25, 533-567.

Wu, S., Bachrach, A., Cardenas, C., \& Schuler, W. (2010). Complexity metrics in an incremental rightcorner parser. In Proceedings of the 48th Annual Meeting of the Association for Computational Linguistics ACL '10 (pp. 1189-1198). Association for Computational Linguistics.

Yadav, H., Vaidya, A., Shukla, V., \& Husain, S. (2020). Word order typology interacts with linguistic complexity: a cross-linguistic corpus study. Cognitive Science, 44.

Yamashita, H. (1997). The effects of word-order and case marking information on the processing of japanese. Journal of Psycholinguistic Research, 26, 163-188.

Yamashita, H. (2000). Structural computation and the role of morphological markings in the processing of japanese. Language and speech, 43, 429-455.

Yngve, V. H. (1960). A model and an hypothesis for language structure. Proceedings of the American philosophical society, 104, 444-466.

Zola, D. (1984). Redundancy and word perception during reading. Perception \& Psychophysics, 36, $277-284$. 